Check for updates

Cite this: Mater. Chem. Front., 2020, 4, 1569

Received 25th December 2019,

Accepted 27th February 2020

DOI: $10.1039 / c 9 q m 00779 b$

rsc.li/frontiers-materials

\section{Aptamer-functionalized nanomaterials for biological applications}

\author{
Qiaoli Ren, $\dagger^{\mathrm{a}}$ Lu Ga, $\dagger^{\mathrm{b}}$ Zhili Lu, ${ }^{\mathrm{c}}$ Jun Ai (D) $*^{\mathrm{a}}$ and Tie Wang (D) *d
}

\begin{abstract}
Aptamers have attracted the attention of many researchers because of their high stability, high affinity and high selectivity. Over the past decades, considerable intensive exploration and research has been carried out in this area. These studies have led to the emergence of functionalized nanomaterials, which have suggested a more diverse range of potential applications. However, despite the many exciting and remarkable developments emerging at the moment, several challenges and issues remain to be addressed. Based on currently reported aptamer-functionalized nanomaterials (AFNs), this review highlights potential applications for AFN platforms in catalysis, detection, cellular analysis, imaging, and targeted drug delivery. We also focus on explaining controllable syntheses and the unique dynamic properties of AFNs. Finally, we discuss progress and the challenges associated with AFN applications in this field.
\end{abstract}

\section{Introduction}

With the rapid development of modern biology in the 20th century, the structures of proteins, antibodies, nucleic acids,

\footnotetext{
${ }^{a}$ College of Chemistry and Environmental Science, Inner Mongolia Normal University, 81 zhaowudalu, Hohhot 010022, People's Republic of China. E-mail: imacaj01@163.com

${ }^{b}$ College of Pharmacy, Inner Mongolia Medical University, Jinchuankaifaqu, Hohhot 010110, People's Republic of China

${ }^{c}$ Key Laboratory of Materials Processing and Mold, Ministry of Education, Zhengzhou Universit, P. R. China

${ }^{d}$ Beijing National Laboratory for Molecular Sciences, Key Laboratory of Analytical Chemistry for Living Biosystems, Institute of Chemistry Chinese Academy of Sciences, Beijing, People's Republic of China. E-mail: wangtie@iccas.ac.cn $\dagger$ These authors contributed equally to this work.
}

polysaccharides, lipids and other macromolecules have been elucidated successively. ${ }^{1-5}$ Follow this trend, the 21st century has been the century of life science. Aptamers are strands of oligonucleotides (RNA or ssDNA) or amino acid fragments with a special tertiary structure, which are selected in vitro from large random libraries by a process called systematic evolution of ligands by exponential enrichment (SELEX). ${ }^{6,7}$ Aptamer of SELEX has a high affinity and specificity to non-nucleic acid targets, such as peptides, proteins, drugs, organic and inorganic molecules, or even whole cells. Nucleic acid aptamers (RNA or ssDNA) have been suggested repeatedly as promising emerging therapeutic agents and recognition elements. A single strand of DNA or RNA, usually 10-100 nucleotide residues, was obtained by amplification or screening from the synthetic DNA/RNA library. Even RNA or ssDNA aptamers acting on the same target have

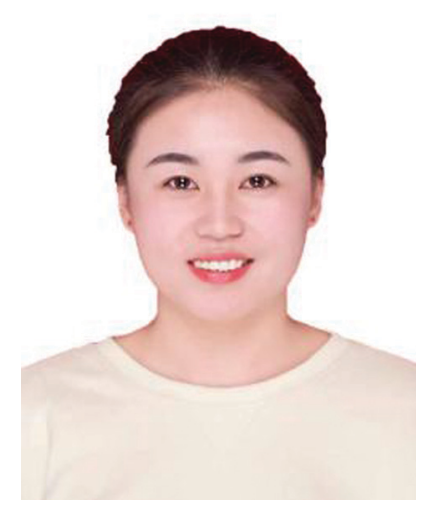

Qiaoli Ren
Qiaoli Ren is currently a Master student at the Inner Mongolia Normal University (with Professor Jun Ai). And during this period, she is also a joint Master student at the Institute of Chemistry, Chinese Academy of Sciences (with Professor Tie Wang). She is currently focused on research into photochromic materials and biosensor construction.

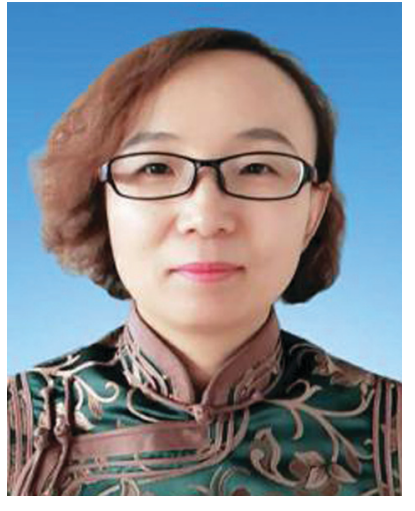

Lu Ga obtained her BSc degree in Chemistry in 2000 and her MS degree in Medical Chemistry in 2005 from Inner Mongolia Normal University and Inner Mongolia Medical University. At present, she is an associate professor and works at Inner Mongolia Medical University. Her current research is focused on Mongolian medical nanomaterials. 
different sequences and tertiary structures. ${ }^{8}$ The chemical properties of aptamers are based on nucleic acid molecules, which has the obvious advantage that they are small in size, non-toxic, easy to synthesize, easy to modify chemically with various functional groups, have low immunity, allow identification of diverse targets, and show easy surface immobilization in biological applications. In fact, aptamers are so superior in terms of their advantages over antibodies that they have been considered ideal substitutes for antibodies. Furthermore, as a new type of functional molecular probe, they have overtaken the decades-long dominant position of antibodies in molecular recognition., ${ }^{9,10}$ Aptamers have found more and more applications in life science, chemistry and other fields, including diagnostic and therapeutic tools, biosensor probes, and drug discovery and release. Of all these areas of research, the field of life science mainly focuses on the treatment and diagnosis of diseases. Researchers are currently trying to develop adaptations that specifically identify targets in diseases such as cancer and viral infections.

Nanotechnology is a type of technology platform that is being more and more widely applied..$^{11-16}$ The term describes the use of technology for design, synthesis, characterization and application at the nanoscale. Control at the nanoscale and manipulation of basic molecular structures allows for the regulation of the macroscopic chemical and physical properties of materials and devices. As the fields of materials science, chemistry and engineering have evolved over the past few decades, nanotechnology is still being developed in all areas where the small scale plays a crucial role in determining fundamental properties. To date, nanotechnology has been widely used in the fields of physics, engineering, chemistry, biology and medicine. ${ }^{17-19}$

With the rapid development of nanotechnology and biotechnology over recent decades, it is now possible to combine these two exciting fields to realize new possibilities. The wide range of biological applications of different nanomaterials in biological analysis, diagnosis and biomedical treatment has aroused great interest. ${ }^{20,21}$ Even though some progress has been reported in the field of aptamer-functionalized nanomaterials (AFNs), this is such a promising field that further effort and exploration is required. This review will systematically explain controllable syntheses, dynamic properties, and potential applications of AFNs. Finally, we will also offer some perspectives on the challenges facing future advancements in this field.

\section{Aptamer-functionalized nanomaterials}

To fully realize the potential of nanomaterials in biological applications, they must be biocompatible and able to target specific biomolecules in order to ensure selective sensing, imaging and drug delivery in complex environments such as living cells, tissues, animals and humans. ${ }^{22}$ The immobilization of functional aptamers to nanoscale or microsized carriers is a powerful way to build new multi-functional materials with desirable properties. In general, nanomaterials can be easily adapted for functionalization through covalent bonding or non-covalent action. ${ }^{23,24}$ This modification aims to improve detection sensitivity and selectivity, reduce analysis time and improve the specificity of binding to target objects. Nanomaterials are commonly used as a substrate for fixing aptamers or as markers for signal amplification because of their ability to absorb cellular components and metabolites, which can be modified by aptamers to cells. Therefore, the use of nanomaterials in medicine represents a breakthrough in the development of materials because the materials are designed to interact with the human body at a cellular scale with a high degree of specificity. Nanomaterials, involving quantum dots, nanoclusters (NCs), nanowires, nanosheets and nanotubes, are characterized by at least one dimension in the nanometer range. ${ }^{25,26}$ (i) Quantum dots have the advantages of traditional organic fluorophores, including light stability, chemical stability, narrower emission spectra and wider excitation spectra. In addition, their surface can be easily modified by chemicals or biopolymers.

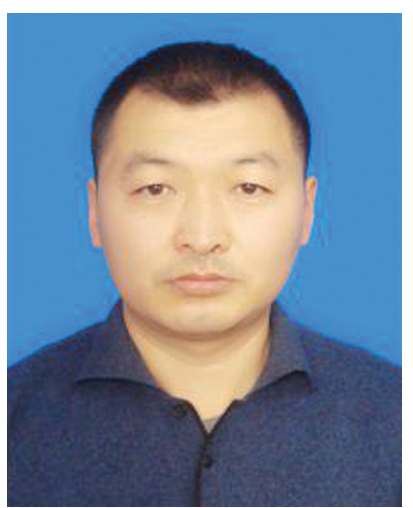

Jun Ai
Jun Ai obtained his BSc and MS degree in Chemistry in 2000 and 2004, respectively, from Inner Mongolia Normal University and Inner Mongolia University. $\mathrm{He}$ then worked in Inner Mongolia Normal University and at present he is a professor. In 2009, he joined Prof. Erkang Wang's group, and received his $P h D$ degree in analytical chemistry in January 2013 from Changchun Institute of Applied Chemistry, Chinese Academy of Sciences. His studies are centered on biosensor construction and fabrication of functional nanobiomaterials.

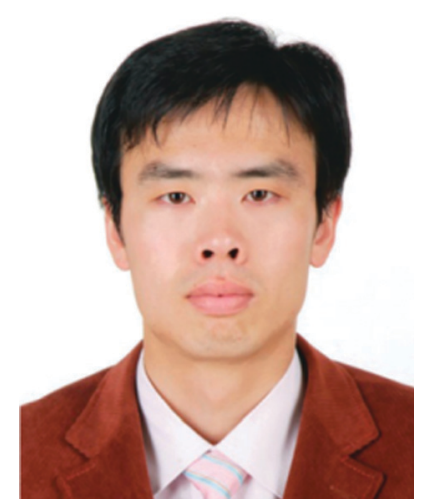

Tie Wang
Tie Wang is a professor at the Institute of Chemistry, Chinese Academy of Sciences (ICCAS). $\mathrm{He}$ received his $\mathrm{BSc}$ degree from $X{ }^{\prime}$ 'an Jiaotong University in 2002 and his $P h D$ degree from Changchun Institute of Applied Chemistry, Chinese Academy of Sciences in 2007. He then worked as a postdoctoral fellow at Rensselaer Polytechnic Institute, Troy, NY, and the University of Florida, Gainesville, FL. He joined ICCAS with the award of the

"Thousand Youth Talents Plan" in 2013. In 2019, he was awarded "outstanding youth of the national fund council". His research focuses on nanoparticle assemblies and their applications. 
This is an advantage that most of bulk materials do not have. (ii) Nanoclusters, such as gold and silver, are a new class of fluorophores. $\mathrm{Au}$ and $\mathrm{Ag}$ NCs have an appealing set of features that complement the properties of organic dyes and quantum dots. They also have desirable photophysical properties and low toxicity suitable for biological applications. (iii) Nanowires typically exhibit better mechanical properties than bulk materials as their size decreases. As size is reduced, strength and toughness become greater. (iv) There are only a few reports on aptamerfunctionalized nanosheets currently, most of which are graphene nanosheets. (v) Nanotubes, such as single-wall carbon nanotubes (SWCNTs), have a high aspect ratio, high surface area and excellent material properties, including electrical conductivity, thermal conductivity and mechanical strength, which offer a wide range of opportunities and potential applications in biology and medicine. Nanomaterials can be broadly divided into two categories: nanoparticles and nanobulk materials. Nanoparticles are microscopic particles at the nanoscale, defined as particles less than $100 \mathrm{~nm}$ in at least one dimension, or materials made of them as basic units. Nanobulk materials are blocks formed by particles with a size of $\sim 1-100 \mathrm{~nm}$. The term describes a one-, two- or three-dimensional systems constructed and composed on a nanoscale as a basic unit according to certain laws, in which the crystal size, crystal boundary width, precipitation phase distribution, pore size and defect size are all of the order of nanometers. Nanoparticles have unique properties compared with bulk materials in terms of optical, thermal, electrical, magnetic, mechanical, and chemical properties. Because their basic structural units are on the nanoscale, nanomaterials have special properties compared with traditional bulk materials, such as quantum size effect, small size effect, surface effect and macroscopic quantum tunneling effect. Furthermore, in the case of DNA, it usually requires the use of cationic transfection agents to achieve cell absorption. ${ }^{27,28}$ However, it is these substances that penetrate into cells making it difficult to perform biological sensing in the living body, and also causing damage to the nucleus. To address this obstacle, nanomaterials, such as metals, carbon, silicon or magnetic materials, have been used as DNA carriers or assistants. By combining the inherent features of nanomaterials with the specific recognition ability of aptamers, a range of nanomaterial-aptamer conjugates have proven their utility in multiple areas. Aptamers can be used to perform a variety of functions, across two main directions: (1) based on their specific binding to target molecules, aptamers have great potential in biomedical applications, such as drug delivery and the development of new therapeutic systems. (2) The properties of aptamer means that they play an important role in the development of new biosensors. So far, nanomaterials such as metals, magnetism, silica, hydrogels and carbon materials have helped many advances in biology. These nanomaterials typically have a large surface area and have unique dimensions and shapes, as well as physical and chemical properties associated with their composition, including surface plasmon resonance (SPR), fluorescence, magnetism and/or load capacity. The cross-linking of these properties has allowed researchers to synthesize AFNs as needed, as summarized in Table 1.

This shows that the two areas of nanotechnology and biotechnology may have broader prospects by complementing each other. To date, noble metal nanoparticles in these nanomaterials have been reported and have shown particular potential in achieving these tasks due to their unique chemical and physical properties, simple synthesis, good biocompatibility and easy integration with biomolecules. It is well known that aptamer-coupled $\mathrm{Au}$ nanomaterials or $\mathrm{Ag}$ provide a powerful platform for targeted recognition, detection and treatment. ${ }^{41-47}$ In 2004, Willner et al. reported that catalytic amplification of aptamer-functionalized $\mathrm{Au}$ nanoparticles (AuNPs) amplifies the optical detection of aptamer-thrombin complexes in solution and on the surface. ${ }^{48}$ Since then, the Liu group were the first to report aptamer-functionalized AuNPs as probes in a dry-reagent strip biosensor for protein analysis. ${ }^{49}$ More recently, $\mathrm{Xu}$ et al. have reported the rapid identification of lipopolysaccharide using the different binding affinities between two aptamer-functionalized AuNPs. ${ }^{45}$

In addition, nanomaterials are fascinating in terms of their composition-, size-, and shape-dependent properties. For example, mesoporous silica nanoparticles (MSN) were designed as promising carriers due to their unique characteristics, such as high loading capacity, biocompatibility, high thermal stability, uniform porosity, adjustable pore size $(2-10 \mathrm{~nm})$, and easy functionalization of internal and external surfaces. ${ }^{50}$ Therefore, silica nanoparticles have also become an important platform for biomedical

Table 1 Specific categories of functionalization of several functionalized nanomaterials

\begin{tabular}{|c|c|c|c|}
\hline Aptamer-nanomaterials & Immobilization and features & Application & Ref. \\
\hline \multirow{3}{*}{$\begin{array}{l}\text { Magnetic nanoparticles } \\
\left(\text { e.g. } \mathrm{Fe}_{3} \mathrm{O}_{4}, g-\mathrm{Fe}_{2} \mathrm{O}_{3}\right)\end{array}$} & \multirow{3}{*}{$\begin{array}{l}\text { Specificity and magnetism } \\
\text { (high throughput separation capabilities) }\end{array}$} & Used as imaging agents & 29 \\
\hline & & Magnetic fluid hyperthermia therapy & 30 \\
\hline & & Drug delivery & 31 \\
\hline \multirow[t]{2}{*}{ Metals (e.g. gold, silver) } & \multirow[t]{2}{*}{ SPR properties } & Any analyte & 32 \\
\hline & & Heterogeneous assays & 33 \\
\hline \multirow[t]{2}{*}{ Silica } & \multirow{2}{*}{$\begin{array}{l}\text { H density and easy separation, } \\
\text { supplied internal doping, biocompatible }\end{array}$} & Drug delivery and oxygen sensing (diagnostics) & 34 \\
\hline & & Targeted recognition and delivery & 35 \\
\hline \multirow[t]{2}{*}{ Hydrogels } & \multirow{2}{*}{$\begin{array}{l}\text { Hold large amounts of water } \\
\text { or biological fluids }\end{array}$} & Molecular recognition and separation & 36 \\
\hline & & Signal transduction and amplification & 37 \\
\hline \multirow{3}{*}{$\begin{array}{l}\text { Carbon materials (e.g. single-walled } \\
\text { carbon nanotubes) }\end{array}$} & \multirow{3}{*}{$\begin{array}{l}\text { High aspect ratio, high surface area, and } \\
\text { excellent material properties, electrical and } \\
\text { thermal conductivity and mechanical strength }\end{array}$} & Field effect transistors & 38 \\
\hline & & Quenchers for fluorophores & 39 \\
\hline & & NIR optical protein assay & 40 \\
\hline
\end{tabular}

NIR, near-infrared; SPR, surface plasmon resonance. 
applications and biological analysis. Dinarvand and co-colleagues developed aptamer-functionalized polyethylene glycol coated superparamagnetic iron oxide nanoparticle (SPION)/mesoporous silica core-shell nanoparticles for simultaneous targeted cancer therapy and magnetic resonance imaging for the development of nanomaterials with thermal diagnostic capabilities. ${ }^{51}$

Precise control the structure of metal nanomaterials is also of great significance for the development of advanced nanobiological technologies. Some assembly techniques have been shown to provide methods for the immobilization of nanoscale particles and to obtain highly dispersed nanomaterials. ${ }^{52,53}$ Countless functional aptamers were constructed by coupling interactions linking the building blocks or by modulating their agreement pattern. The breakthrough related to the assembly of functionalized nanomaterials with synthetic aptamers which allowed a series of new physical and chemical properties to emerge, including mechanical, wetting, optical, magnetic and electrical properties. Therefore, the functional performance of AFN assemblies has allowed practical applications to be expanded and improved. ${ }^{54}$

Numerous studies have shown that aptamers, DNA/RNAzymes or aptazymes can generally induce assembly of nanomaterials. ${ }^{55-58}$ To date, the DNA molecule is probably the most widely used novel biomolecular material designed to regulate structure and self-assemble. DNA-based sequence-oriented functional features may include specific recognition properties, catalytic functions, reactions to enzymes or sequence-specific binding of proteins; these unique functional oligonucleotides are widely used to develop DNA-based sensors, construct DNA machines and assemble DNA nanostructures. ${ }^{59}$ Some studies have shown that DNA can mediate surface functionalization, shape-controlled synthesis, and collaborative assembly of nanoparticles, such as specific biological probes. In 2013, two different self-assembly methods for the preparation of red or yellow nucleic acid stable Ag NC nanowires were reported. ${ }^{60}$ As shown in Fig. 1, they used two different approaches to prepare functional template nanowires, including the hybridizationpolymerization reaction or the hybridization chain reaction. This study confirmed that DNA provides a functional template for the deposition of orderly and stable Ag nanowires, which led to the production of DNA nanowires, which have adjustable luminescence functions. This research laid the foundation for the development of multiple DNA (or aptamer) analysis schemes based on luminescent nucleic acid stable Ag NC nanowires, and other sequence-specific metals (e.g., $\mathrm{Au}, \mathrm{Cu}$ ) can also be implemented. In addition, one study recently described doublestranded DNA molecules (dsDNA) designed to be anchored on AuNPs to regulate the crystallization of gold atoms from the surface of AuNPs to the end of DNA; mainly driven by the distribution gradient of the gold precursor. Furthermore, starlike AuNCs (nanometer stars) can be synthesized by single or multiple branches under the guidance of linear dsDNA. ${ }^{61}$ This study reports that DNA, as a molecular regulator of nanocrystalline growth, makes possible the synthesis and design of metal nanocrystalline in biomedical imaging, optical antenna and biomolecular sensors. Modification of nanoparticles by specific aptamers has advantages in different application fields. The properties of aptamer-modified nanoparticles can be used in biosensors. If cell-specific aptamers are used, the resulting conjugates can be used for cell targeting, targeted drug delivery and so on.

\section{Properties of aptamer- functionalized nanomaterials}

Systems based on AFN composites not only have the dual properties of aptamers and nanomaterials, but also have many new properties, such as high affinity, strong degradation and high cell uptake between functionalized nanomaterial complexes and targets. The properties of these functionalized nanomaterials may play a key role in biomedical applications; for example, the efficient self-delivery to cancer cells is the first condition for exploring the intracellular environment. ${ }^{62,63}$

\subsection{Large specific surface area and abundant, easily accessed metal sites}

With decrease in particle size, the specific surface area of nanomaterials increases greatly, and the number of surface atoms has also increased substantially as a proportion of the total number of particles. An increase in the total number of surface atoms will lead to an increase in the coordination unsaturated properties of surface atoms, which will result in a large number of suspended bonds and unsaturated bonds. Surface defects also increase. Therefore, nanomaterials have higher catalytic activity compared with large-scale catalysts. Gold, for example, has long been considered a low-activity catalytic material, but when gold is dispersed to the nanometer level, it can show high catalytic activity. AFNs also possess these properties, having a large surface area and depending on the unique size and shape, as well as the physical and chemical properties of the composition. ${ }^{64}$ Many different materials can attach biomolecules, such as aptamers, through a variety of surface chemical reactions. One reason for the specific fixation of the aptamer on the surface of the nanostructure may also be that the large surface area of the material increases the density of the aptamer on the material, thus increasing the area of interaction between the aptamer and the target analyte. Another major reason is that the combination of the intrinsic properties of nanostructured materials with the binding properties of immobilized aptamers provides possibilities for a variety of applications.

\subsection{Conformational flexibility}

In general, aptamers can change their secondary structure after being combined with the target, folding into a unique secondary or three-dimensional structure, giving them high affinity and specific binding abilities toward their corresponding targets. ${ }^{65}$ Target binding can induce significant conformational changes in aptamers, and based on these conformational changes, targets can be detected. Many efforts have been made to design and build 
a
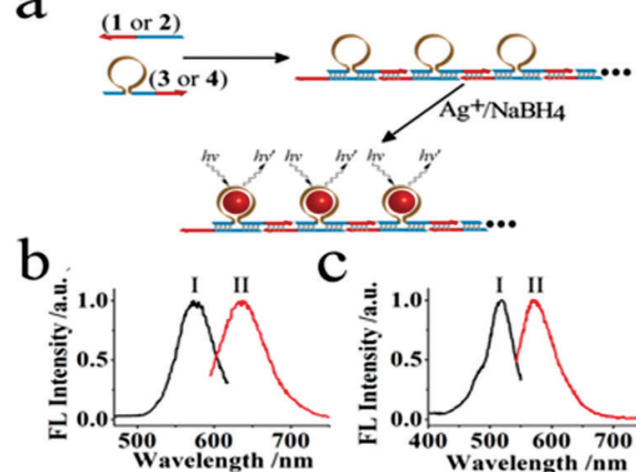

C

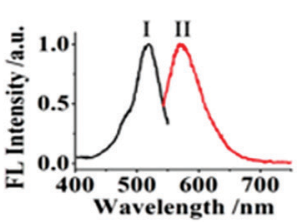

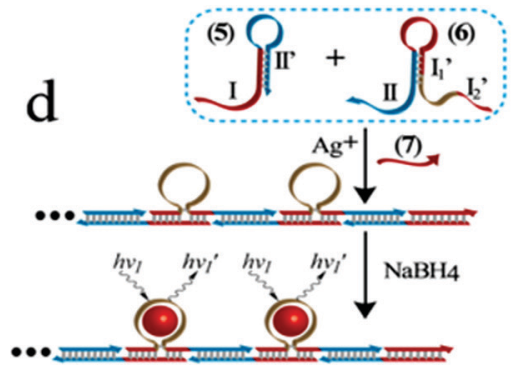

e

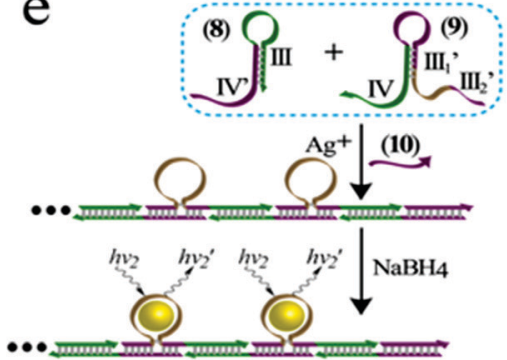

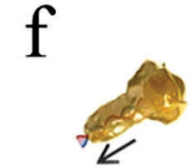

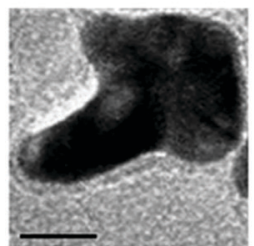

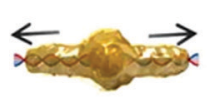

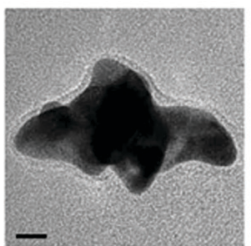

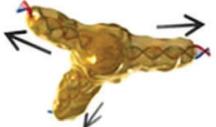

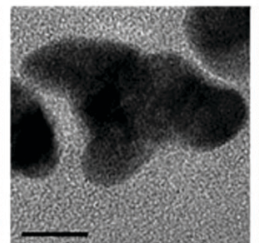

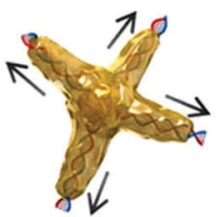

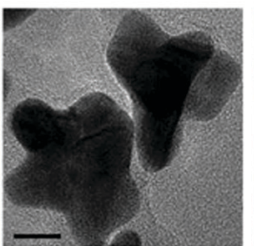

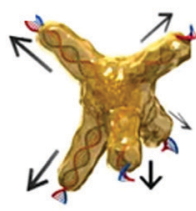

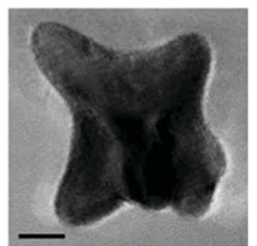

Fig. 1 (a) Autonomous hybridization-polymerization process that leads to chains consisting of sequence-specific nucleic acid hairpin structures on DNA scaffolds for the deposition and stabilization of luminescent AgNCs. (b) Excitation (I) and luminescence (II) spectra of the red-emitting AgNCs nanowires. (c) Excitation (I) and luminescence (II) spectra corresponding to the yellow-emitting AgNCs nanowires. Autonomous nucleic acid (target)induced activation of the hybridization chain reaction that involves cross-opening of two functional hairpin structures that lead to DNA chains, consisting of sequence-specific hairpins on DNA scaffolds that stabilize the (d) red- or (e) yellow-emitting AgNCs. Image reprinted from ref. 60 with permission. Copyright 2013 American Chemical Society. (f) Illustrations and corresponding transmission electron microscopy (TEM) images of star-shaped AuNCs with controlled branches from 1 to 5, respectively. Scale bars, $10 \mathrm{~nm}$. Image reprinted from ref. 61 with permission. Copyright 2016 Nature.

various logic gates. Based on the conformational flexibility, this has led to a breakthrough that enables most logic devices that could previously perform only one operation to perform the operation of multiple universal logic devices. Our group hypothesized that two or more different sizes of fluorescent transmitters can be formed by undergoing structural changes in a single DNA template in some cases. We reported cationic $\mathrm{K}^{+}, \mathrm{H}^{+}$, which induced a hair clip DNA template (called HP26) into structural change, regulating the preparation HP26-stabilized Ag fluorescence behavior as shown in Fig. 2. ${ }^{66}$ This study suggested the idea of constructing more logic devices by changing the specific sequence of DNA stabilizers to construct logic devices.

\subsection{High affinity and specificity}

The high affinity and specificity of aptamers toward targets is generally achieved by a combination of molecular shape complementarity, hydrogen-bonding interactions, and stacking interactions. ${ }^{67}$ Based on the specific binding properties of an aptamer, Yao and co-workers reported a method used to detect three different chemicals; adenosine, dopamine and $17 \beta$-estradiol. ${ }^{68}$ In the study, Ru complexes and quantum dots (QDs) were used as fluorescent probes. Ru complexes can quench the fluorescence of QDs, and QDs also preferentially bind to the adaptive DNA, resulting in an increase of the fluorescence of QDs. When the aptamer is specifically bound to the target, the Ru complex is in an unbound state, so the fluorescence of QDs is quenched. This method has the advantages of requiring no label, having low cost, high selectivity and repeatability, which make it significant.

\subsection{Biocompatibility}

Biocompatibility is the degree that a material is compatible with the human body after implantation. That is, whether it will cause toxic effects on human tissues. ${ }^{69}$ Biomaterials are an 

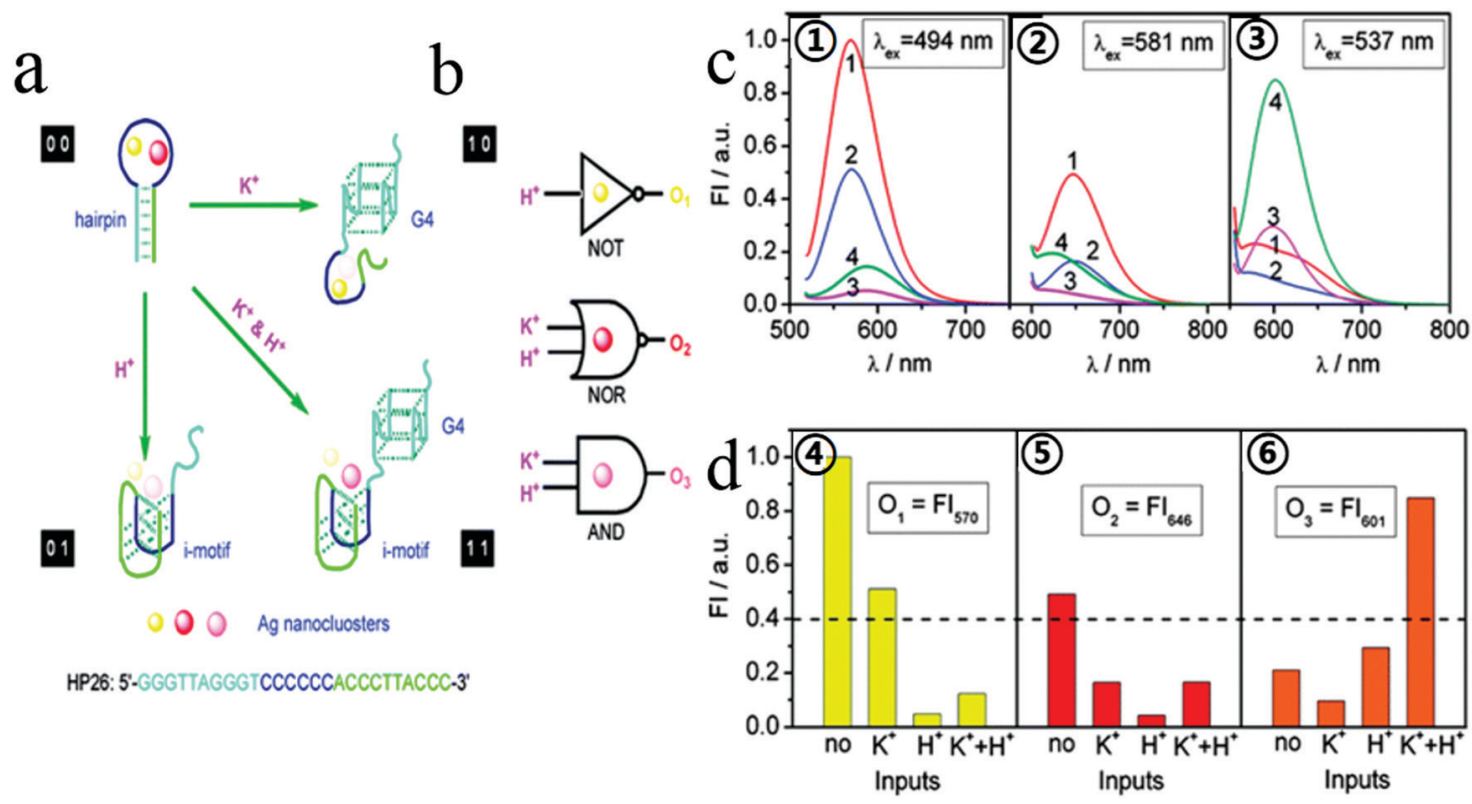

Fig. 2 (a) Schematic diagram of logic operations based on HP26-tuned fluorescent Ag NCs, with corresponding symbols of logic gates. $\mathrm{K}^{+}$and $\mathrm{H}^{+}$serve as two inputs to trigger the allosterism of HP26 and modulate the fluorescence output. (b) HP26-stabilized Ag NCs for multiple logic operations. (c, (1)-3) Fluorescence emission spectra of Ag NCs at different excitation wavelength in $10 \mathrm{mM}$ tris-Ac buffer $(\mathrm{pH}=8.0)$ at four input modes: 1 , no input; 2100 mM $\mathrm{K}^{+} ; 3, \mathrm{H}^{+}(\mathrm{pH}=5.0) ; 4, \mathrm{~K}^{+}$and $\mathrm{H}^{+}$. (d, (4)-(6) Bar representations of the fluorescence intensity at $570 \mathrm{~nm}, 646 \mathrm{~nm}$ and $601 \mathrm{~nm}$. The same threshold value (0.4) for output 1 or 0 is set at all fluorescence channels. Image reprinted from ref. 66 with permission. Copyright 2011 American Chemical Society.

important technology, allowing the development of tissue engineering, while their respective biocompatibility and host reaction are key factors for the use of biomaterials in tissue engineering. However, because important concepts around biological reactions, infections, surface interactions and material properties are still poorly understood, this limits the application of medical materials. Therefore, biocompatibility is urgently needed for further development, not only because of its importance in interdisciplinary science but also because of its importance in medicine and various other applications. $^{70}$ In addition, materials interact with tissues through cell adhesion, and the biocompatibility of materials is also closely related to cell adhesion. ${ }^{71}$ Therefore, the first problem to be solved in developing new materials and modifying surfaces to create devices that are responsive to the environment is surface biocompatibility. At present, many methods of improving biocompatibility by surface modification have been reported. but there are still many challenges to overcome. Surface biocompatibility is important; for example, the properties of bulk materials need to be carefully considered when selecting biomaterials for specific purposes. Other functions such as self-cleaning, intelligent healing, self-repair, control of degradation and function are also important. In addition, the toxicity of these materials requires further study.

\section{Applications of aptamer- functionalized nanomaterials}

Due to the unique size and structure of functionalized nanomaterials, they have many physical and chemical properties that traditional materials do not, including a surface effect and small size effect, quantum size effect and macroscopic quantum tunneling effect, for example. Combining nanomaterials with aptamers can effectively improve selectivity and sensitivity of AFNs.

\subsection{Catalysts}

Some molecular recognition components, generally including nucleic acid enzymes and proteins, have catalytic properties. For example, catalytic DNA is a kind of functional DNA with catalytic properties. These components can catalyze different kinds of chemical and biological reactions, including cleavage of nucleic acid substrates, small molecule decomposition, nucleic acid ligation, nucleic acid structure rearrangement and porphyrin metalation. Because of its unique catalytic properties and extensive catalytic action, catalytic DNA has been widely used in biosensors, disease diagnosis and nanostructure assembly.

DNAzyme is one of the most widely used synthetic functional nucleic acids, which can simulate the catalytic activity of enzymes, and had attracted extensive research in the past 20 years. To date, several strategies for the regulation of catalytic DNA activity have been proposed. ${ }^{72}$ Guo et al. reported thermosensitive catalytic systems in which the surfaces of AuNPs are simultaneously modified with a thermoresponsive polymer, poly( $N$-isopropylacrylamide) (pNIPAM), and catalytic DNA. ${ }^{73}$ The system is designed to regulate the catalytic activity of DNA by changing the temperature. The results show that the catalytic activity in the designed catalytic system is greatly affected by temperature. A thermosensitive pNIPAM on the surface of AuNPs enables controlled catalytic activity over a narrow temperature range. The catalytic DNA/pNIPAM/AuNP 
system has little catalytic activity at temperatures lower than the PNIPAM critical solution temperature (LCST). When the temperature rises above LCST, it shows high catalysis.

However, the relatively high cost of DNAzymes limits its broad potential applications, particularly in everyday sensing applications and in mass production based on DNAzyme catalysis. In addition, most DNAzyme-based sensing or catalytic systems require homogeneous reactions in aqueous solutions, which makes the recovery of these biocatalysts difficult. Therefore, the development of renewable and recyclable DNAzyme-based catalysts is of great significance. Based on this vision and previous works, the Guo group also reported high catalytic and recyclable DNAzyme functionalized pNIPAM microgels, prepared by one-step precipitation polymerization. pNIPAM/DNAzyme microgels have high catalytic activities in aqueous solution at room temperature. ${ }^{74}$ When the temperature is higher than the critical solution temperature of pNIPAM, the catalyst will become hydrophobic and separate from the reaction mixture, which is conducive to the recovery of the catalyst. This strategy will pave the way for building low-cost, high-performance DNA biocatalysts for biometrics and biosensors, and may help in the development of low-cost DNAbased catalysts and purification technologies. As shown in Fig. 3, these pNIPAM/DNAzyme microgel catalysts maintained an initial catalytic activity of $80-91 \%$ after eight cycles.

In addition, DNA also has been reported as a template. The Huang group developed a simple, one-step strategy for the rapid synthesis of DNA template copper nanoparticles (Apt-CuNPs), showing selective targeting and high catalytic activity of peroxides, and it can be used to detect thrombin as shown in Fig. 4. ${ }^{75}$ Interestingly, Apt-CuNPs were rapidly oxidized to Apt-CuO/ $\mathrm{Cu}_{2} \mathrm{O}$ NPs, showing intrinsic peroxidase activity in the presence of $\mathrm{H}_{2} \mathrm{O}_{2}$. Using Mucin-1 binding aptamer-templated $\mathrm{CuO} / \mathrm{Cu}_{2} \mathrm{O}$ NPs, they finally applied the sensing platform to detect Mucin-1-overexpression in tumor cells. This study suggests that the $\mathrm{Apt}-\mathrm{T}_{n}$ can be used as a universal template for the preparation of enzyme-like $\mathrm{CuO} / \mathrm{Cu}_{2} \mathrm{O}$

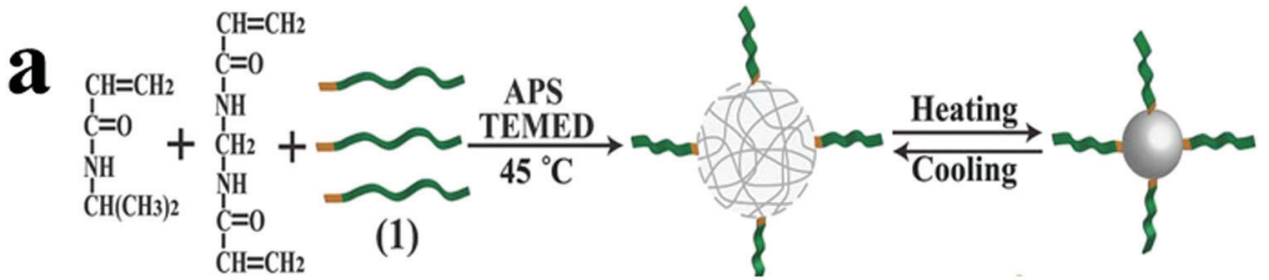

b
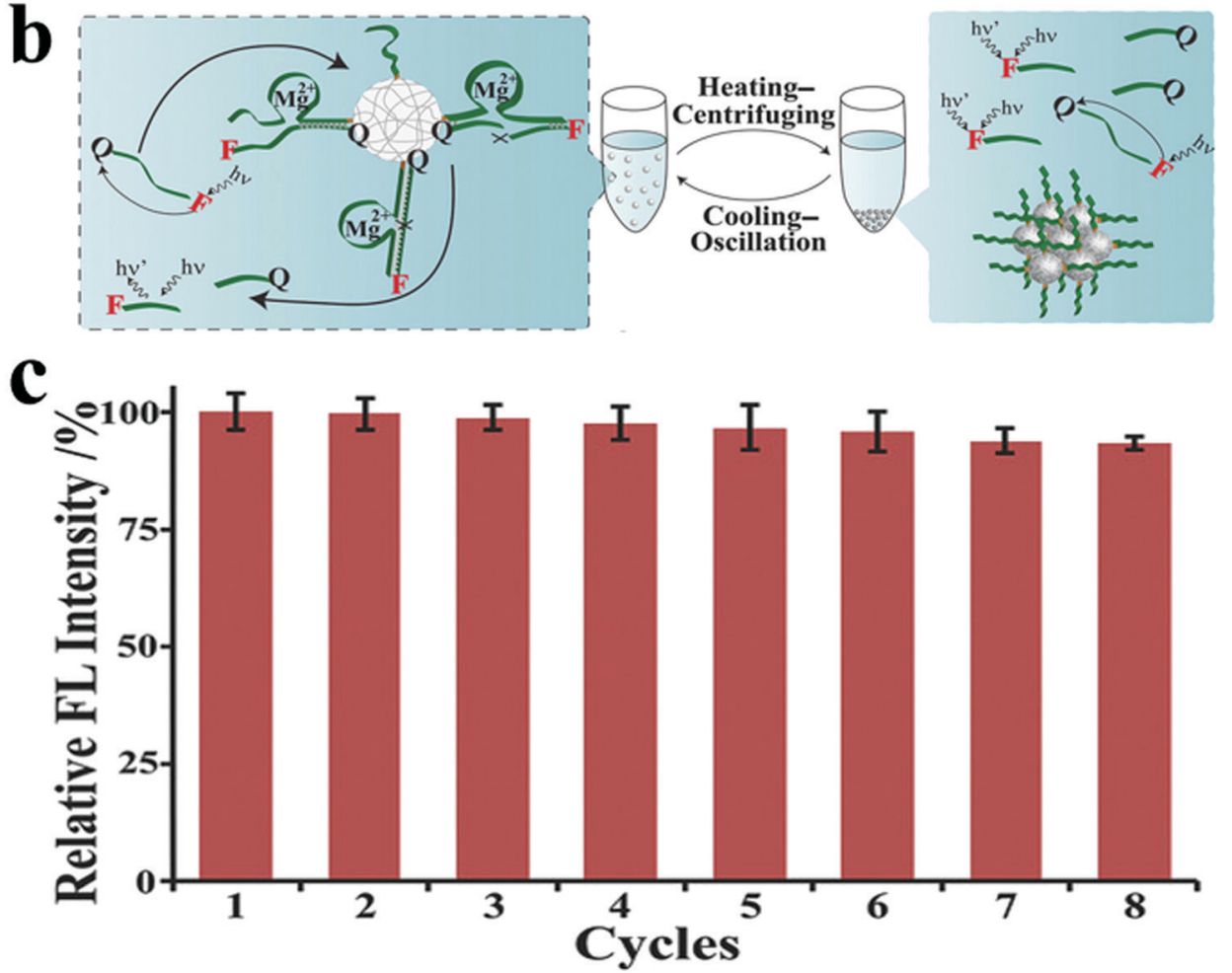

Fig. 3 (a) Schematic illustration of the synthesis of DNAzyme (1) functionalized pNIPAM microgels by redox initiated precipitation polymerization at $45^{\circ} \mathrm{C}$ and the thermoresponsive behavior of DNA/pNIPAM microgels. (b) Schematic diagram for the recycling of pNIPAM/DNAzyme microgels by heating-centrifuging $\left(40^{\circ} \mathrm{C}\right)$ and cooling-oscillation $\left(25^{\circ} \mathrm{C}\right)$ cycle. (c) The catalytic activities of pNIPAM/DNA microgels at $1 \times 10^{-6} \mathrm{M}$ DNA substrate for eight consecutive recycling procedures (relative $\mathrm{FL}$ intensity $=F / F_{\text {cycle1 }} \times 100 \%$ ). Image reprinted from ref. 74 with permission. Copyright 2018 Wiley Online Library. 


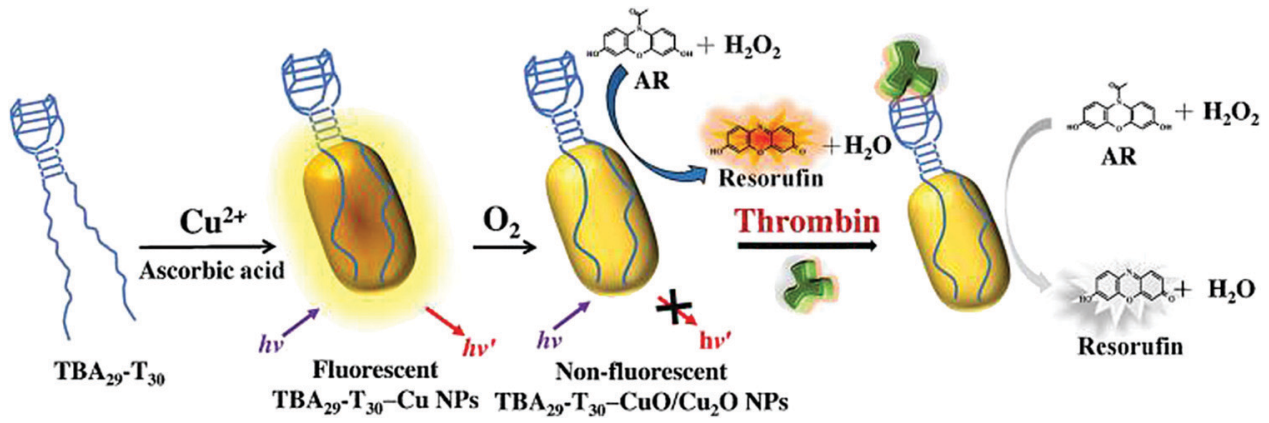

Fig. 4 Schematic representation of the preparation of Apt- $\mathrm{CuO} / \mathrm{Cu}_{2} \mathrm{O}$ NPs exhibiting peroxidase-like catalytic activity, and their application in the determination of thrombin based on inhibition of its enzymatic activity using $\mathrm{H}_{2} \mathrm{O}_{2} /$ AR redox system as fluorescent transducer. Image reprinted from ref. 75 with permission. Copyright 2015 Elsevier.

NPs for the detection of proteins and the labeling of tumor cells when using appropriate aptamers.

\subsection{Imaging}

In order to understand cell function, pathology, disease diagnosis and drug discovery, it is important to be able to monitor the distribution of biomolecules within cells. Cell imaging is a useful approach in understanding the spatial distribution of biomolecules in cells. Imaging using nanomaterials as contrast agents visualizes structures and helps clinicians describe and recommend appropriate treatments. ${ }^{76,77}$ We previously reported the water-soluble DNA encapsulated Ag NCs with near-infrared (NIR) fluorescence, which can be used for simultaneous targeted cancer imaging and enhanced photothermal therapy (PTT) or photodynamic therapy (PDT) due to the synergistic effects between the photodynamic agent and NIR Ag NCs. ${ }^{78}$ In the study, AS1411 was reasonably connected with the DNA scaffold of the Ag NC, retained its secondary structure, and effectively loaded photosensitizer. The binding affinity of AS1411 to the nucleolin with high fluorescence of Ag NCs provided opportunities for their application in the intracellular imaging and nuclear staining. In addition, Li et al. reported the reconfigurable bioinspired framework nucleic acid (FNA) nanoplatform, dynamically manipulated in living cells for subcellular imaging. ${ }^{79}$ They built an FNA logic device on the nanoscaffold of a DNA triangular prism. By using an i-motif and an ATP-binding aptamer (ABA) incorporated into the DTP scaffold, this FNA device logically responds to the changes of lysosomal $\mathrm{pH}$ and ATP levels modulated by external stimuli. Once two tetrahedral DNA nanostructures enter the acidic lysosomes of living cells, they assemble into a heterodimer structure to form a larger framework, which allows subcellular imaging under the action of endogenous ATP. Recently, the authors also reported a lysosomal recognition FNA nanometer device as shown in Fig. 5. ${ }^{80}$ The i-motif and ATP-binding aptamers bind to the DNA triangular prism as logical control units. Once inside the lysosomal vesicles, FNA devices respond to lysosomal $\mathrm{pH}$ and ATP by folding i-motif and ATP-binding aptamers and inducing structural changes in FNA and releasing the reported structures of subcellular imaging. This work addresses the challenge of how to control their activation within a specific cell interval.
Cell-type-specific imaging is also reported in intracellular imaging. Zhu et al. propose a simple one-pot method for the synthesis of AS1411 functional AgNCs with good fluorescence properties as shown in Fig. $6 .{ }^{81}$ They demonstrated that AS1411 functionalized AgNCs could be internalized into MCF-7 human breast cancer cells and specifically stain the nuclei red. Due to the simple synthesis process and the ability to identify specific targets, this fluorescent platform has the potential to expand the application of AgNCs in biosensors and bioimaging.

Aptamers have been proven to be promising molecular probes for in vivo cancer imaging. However, the application of aptamers in vivo may be limited by the lack of physiological temperature affinity in human plasma and the degradation of aptamer nucleases; some aptamers-functionalized nanomaterials also have been developed for in vivo imaging. Zhang et al. assembled a three-way junction (3-WJ) pRNA-human epidermal growth factor receptor $2\left(\mathrm{HER}_{2}\right)$ apt-siMED1 nanoparticle to target $\mathrm{HER}_{2}$-overexpressing human breast cancer via an $\mathrm{HER}_{2}$ RNA aptamer to silence MED1 expression, and found that these ultra-compact RNA nanoparticles were very stable under RNase A, serum and $8 \mathrm{M}$ urea conditions. ${ }^{82}$ In in situ xenograft mouse models, these biosafety nanoparticles effectively target and penetrate tumors that overexpress $\mathrm{HER}_{2}$ after systemic administration.

\subsection{Pesticide detection}

The application of traditional analytical techniques is limited due to the long time consumption, large reagent consumption, complex sample preparation and expensive instrumentation. Therefore, it is of great importance to realize a sensitive, fast and simple detection method. Biosensors with an adaptive recognition unit are called aptamer sensors. Since the aptamer has many unique features, such as unique characteristics and chemical structure, they are widely used to detect biomarkers or factors associated with food, medicine, disease and cancer cells in analytical fields, and are an ideal choice for analysis equipment and technology. Aptamers can bind to different targets such as metal ions, small molecules, proteins, live cells, viruses and bacteria with high affinity and specificity. The use of nanomaterials that are functionalized with aptamers for the selective and sensitive detection of analytes such as small 


\section{a}

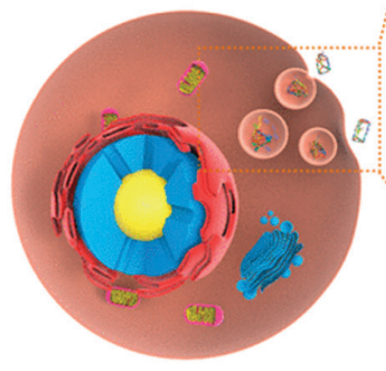

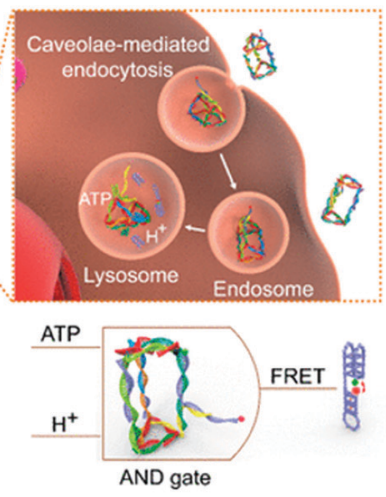

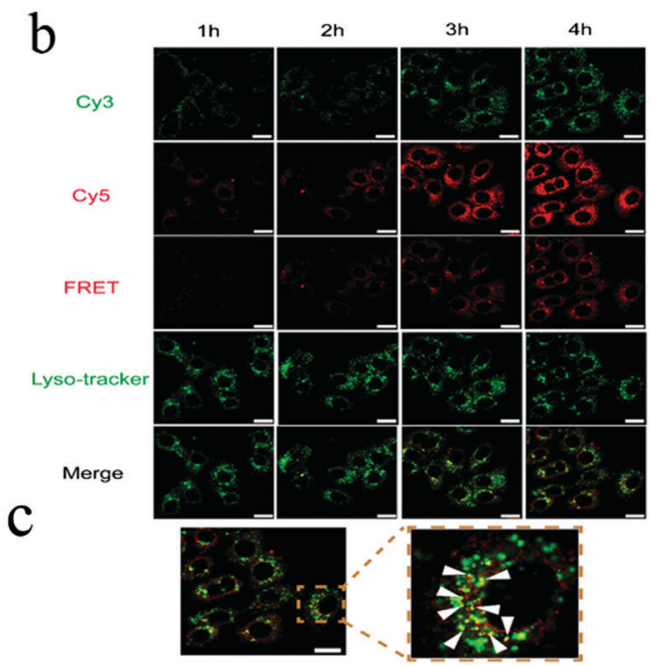

Fig. 5 (a) Schematic illustration of design and working principle of the FNA logic device. (b) Confocal laser scanning microscopy images of MCF-7 cells after incubation with PM4S10 at $37^{\circ} \mathrm{C}$ for 1-4 h. (c) Yellow fluorescence signal demonstrates the colocalization of the red fluorescence resonance energy transfer (FRET) fluorescence signal and the green lyso-tracker fluorescence signal. Scale bars: $20 \mu \mathrm{m}$. Image reprinted from ref. 80 with permission. Copyright 2019 American Chemical Society.
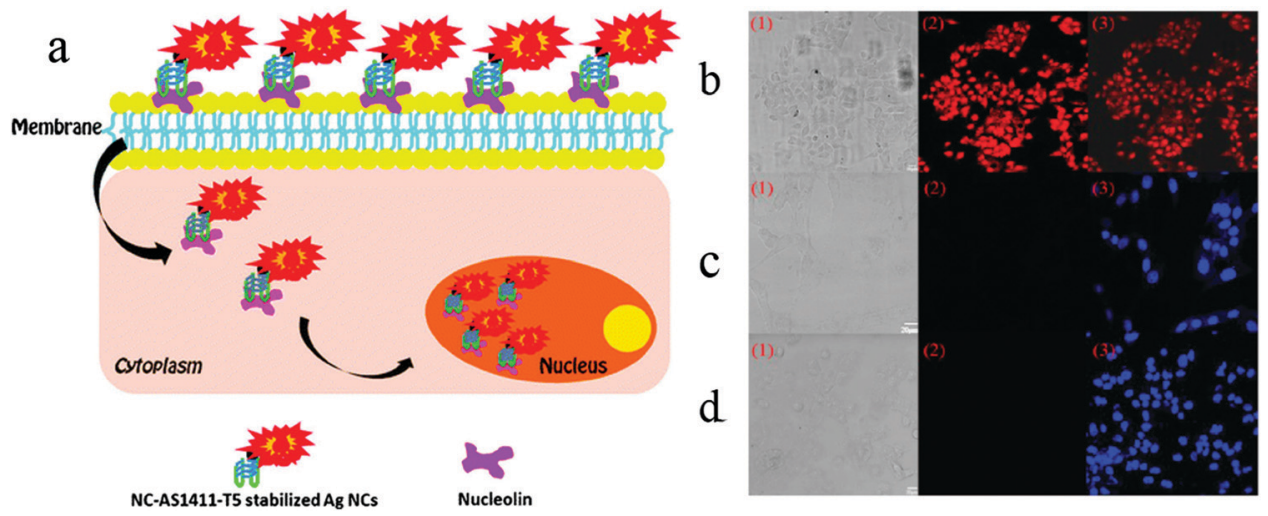

Fig. 6 (a) Schematic illustration of cell-type-specific imaging. (b) Confocal laser scanning microscopy of MCF-7 human breast cancer cells, NIH-3T3 mouse fibroblast cells, and MCF-10A human normal mammary epithelial cells incubated with NC-AS1411-T5-stabilized AgNCs at $4{ }^{\circ} \mathrm{C}$ for 30 min: (1) bright-field images; (2) AgNCs fluorescence images (red); (b3) overlap of corresponding fluorescence image and bright-field image; (c3 and d3) fluorescence images with DAPI nuclear staining (blue). The AgNCs were excited with $543 \mathrm{~nm}$ and DAPI with UV. Scale bar, $20 \mu \mathrm{m}$. Under the same procedure, there were no detectable emissions from the control cells. Image reprinted from ref. 81 with permission. Copyright 2012 American Chemical Society.

molecules, metal ions, proteins, and cells has been demonstrated. ${ }^{83-85}$ Nanomaterials add signal transduction, as well as signal amplification. Some researchers have proposed that the use of multiple molecules (aptamers, small molecules, polymers, and biopolymers) to prepare functionalized nanomaterials should be a worthwhile attempt to overcome nonspecific interactions of pristine aptamers and stabilize functionalized nanomaterials while maintaining their biological functions. ${ }^{86}$ Replacement of nucleic acid aptamers or DNA sequences may lead to the application of this sensing strategy for different target detection. Lin and co-workers reported a chemiluminescence assay with dual signal amplification, which was developed based on multi-DNAzymes-functionalized AuNPs using in situ rolling circle amplification (RCA) for the ultrasensitive detection of thrombin on a microchip. ${ }^{87}$ The linear range of thrombin chemiluminescence detection was good, ranging from 1 to $25 \mathrm{pm}$, and the detection limit was as low as $0.55 \mathrm{ppm}$. Furthermore, multi-channel microchips improve the reaction efficiency and shorten the detection time. Lu et al. reported biosensing based on label-free aptamers and AuNPs for the detection of Escherichia coli (E. coli) O157:H7 and Salmonella typhimurium. The target bacteria binding aptamer was adsorbed on the unmodified AuNP surface to capture the target bacteria. The detection was carried out by inducing the appropriate aggregation of the target bacteria. The color of the aptamer changed from red to purple under high salt conditions. ${ }^{88}$ Pollution due to pesticides has caused wide public concern, because of the extensive use of pesticide in agriculture in order to increase crop yields and improve the quality of agricultural products. The biosensors can achieve the highly sensitive and rapid detection of pesticides without complex sample 


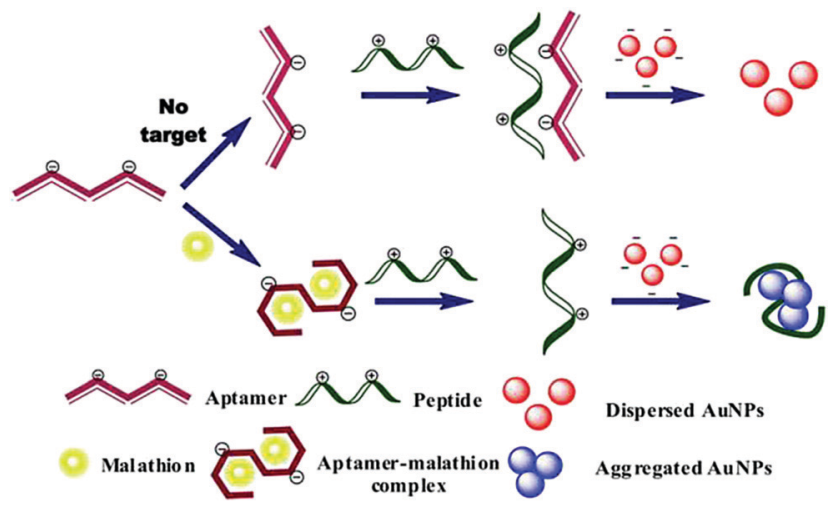

Fig. 7 Schematic illustration of colorimetric assay for malathion based on aptamer, cationic peptide and AuNPs. The AuNPs remain red in the absence of malathion due to the interaction of aptamer and peptide, whereas in the presence of malathion, the color of the particles becomes blue as the aptamer binds to malathion and the peptide is free to aggregate the particles. Image reprinted from ref. 91 with permission. Copyright 2016 Elsevier.

preparation. ${ }^{89,90}$ Over the past few decades, a great deal of work has been done to develop qualitative/quantitative methods for the detection of chemical contaminants in food. The Wangoo group reported a study using aptamers, cationic peptides and unmodified AuNPs to detect malathion, a toxic organophosphorus pesticide widely used in agriculture, as shown in Fig. $7 .^{91}$ When attached to the appropriate body, the peptide disassociated the AuNPs, giving them a red color. However, when the aptamer binds to malathion, the peptide can still be used to cause the aggregation of nanoparticles and turn the suspension blue. The method used optical changes of AuNPs to detect malathion colorimetry. It was found that the method was linear, with a detection limit of $1.94 \mathrm{pM}$ in the range of $0.01-0.75 \mathrm{nM}$.

\subsection{Lab on a chip}

In situations where medical diagnosis and food safety require quick and on-site results, electrochemical biosensors and associated on-chip laboratory equipment are the preferred analytical systems for environmental protection, process control, wastewater treatment and life science discovery research. ${ }^{92}$ To date, reports have also highlighted that lab-on-a-chip (LOC) biosensors have great application potential because they can be miniaturized and automated. They also have the potential to be fast and very sensitive. Some samples may require a more complex preparation process in order to separate or enrich the target analytes with a minimum of distractors before reaching the sensor unit, ultimately improving the analytical performance. LOC is a platform that allows integration of these modules, providing a robust automation system for the required applications. Because a LOC system can perform the complete analysis of a sample, the components of each system are selected based on the analysis. LOC systems typically contain microfluidic channels for sample transport. On the basis of analyzing the problem, the microfluidic system and LOC system are combined. The most important characteristics of the analytical LOC system are the sample preparation, separation and detection.
Recently, Primiceri et al. reported that biochips can be used to quantify two of the most common food-related pathogens; Listeria monocytogenes and Staphylococcus aureus. They all come from suspension of bacterial stationary phase broth culture. The detection limit of $L$. monocytogenes was $5.00 \mathrm{CFU} \mathrm{ml}^{-1}$, and the detection limit of $S$. aureus was $1.26 \mathrm{CFU} \mathrm{ml}^{-1} .{ }^{93}$ Their platform could be a promising real-time medical device for clinical and food diagnostics, but also for biosafety purposes. Malhotra et al. reported the preparation of a label-free chip impedance laboratory (iLOC) for low density lipoprotein (LDL) detection from protein (bovine serum albumin) and an apolipoprotein-B-functionalized carbon nanotube nickel oxide (CNT-NiO) nanocomposite, as shown in Fig. $8 .^{94}$ The cytotoxicity of CNTs, NiO nanoparticles and CNT-NiO nanocomposites synthesized in the presence of lung cancer A549 cell lines was studied using the 3-(45-dimethylthiazol-2-yl)-25-diphenyl tetrazolium bromide (MTT) method. CNT-NiO nanocomposites showed higher cell viability at concentrations of $6.5 \mathrm{~g} \mathrm{ml}^{-1}$ compared with those using single CNTs. Cell viability and proliferation studies showed that the toxicity of CNTs increased with concentration. This iLOC shows excellent sensitivity of $5.37 \mathrm{k} \Omega(\mathrm{mg} \mathrm{dL})^{-1}$ and a low detection limit of $0.63 \mathrm{mg} \mathrm{dL}^{-1}$ in

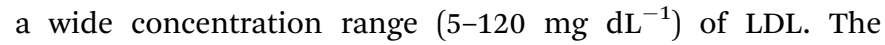
binding kinetics of antigen-antibody interaction of LDL molecules reveal a high association rate constant $\left(8.13 \mathrm{M}^{-1} \mathrm{~s}^{-1}\right)$. Thus, this smart nanocomposite (CNT-NiO)-based iLOC has improved stability and reproducibility, which has implications toward in vivo diagnostics.

\subsection{Nanomedicine}

Nanomaterials can protect the aptamer from the digestion of nucleases, so the AFNs can be used as drug or gene carriers. Drug-carrying nanoparticles can sustain drug release, prolong drug action time and improve target delivery. ${ }^{95}$ They can even improve the target location effect. ${ }^{96}$ Compared to bulk materials, nanoparticles are more likely to penetrate membrane cells and travel along nerve cell synapses, blood vessels, and lymphatic vessels. At the same time, nanoparticles accumulate selectively in different cells and certain cell structures. The use of nanoparticles for highly permeable drugs provides effectiveness.

Drug delivery is an important part of medical treatment. Compared with AFNs, aptamers without modification are more easily degraded by nucleases in vitro, and as a small molecule, it is easily filtered by the kidneys. By loading the nanomaterials, it can overcome the inherent shortcomings of these aptamers. Most modifications to the aptamers aim to improve affinity, potency and metabolic stability. ${ }^{97}$ A key attribute of therapeutic aptamers is the ability to adjust pharmacokinetic characteristics by regulating metabolic stability and by adjusting renal clearance and distribution rates. There have been several reviews on the discovery of aptamers, their general pharmacological properties, and even recent advances in improving the properties of aptamers. ${ }^{98-103}$ Among them, AFNs are being developed as a promising platform for targeted therapy and diagnosis, finding a way to overcome the problem. 


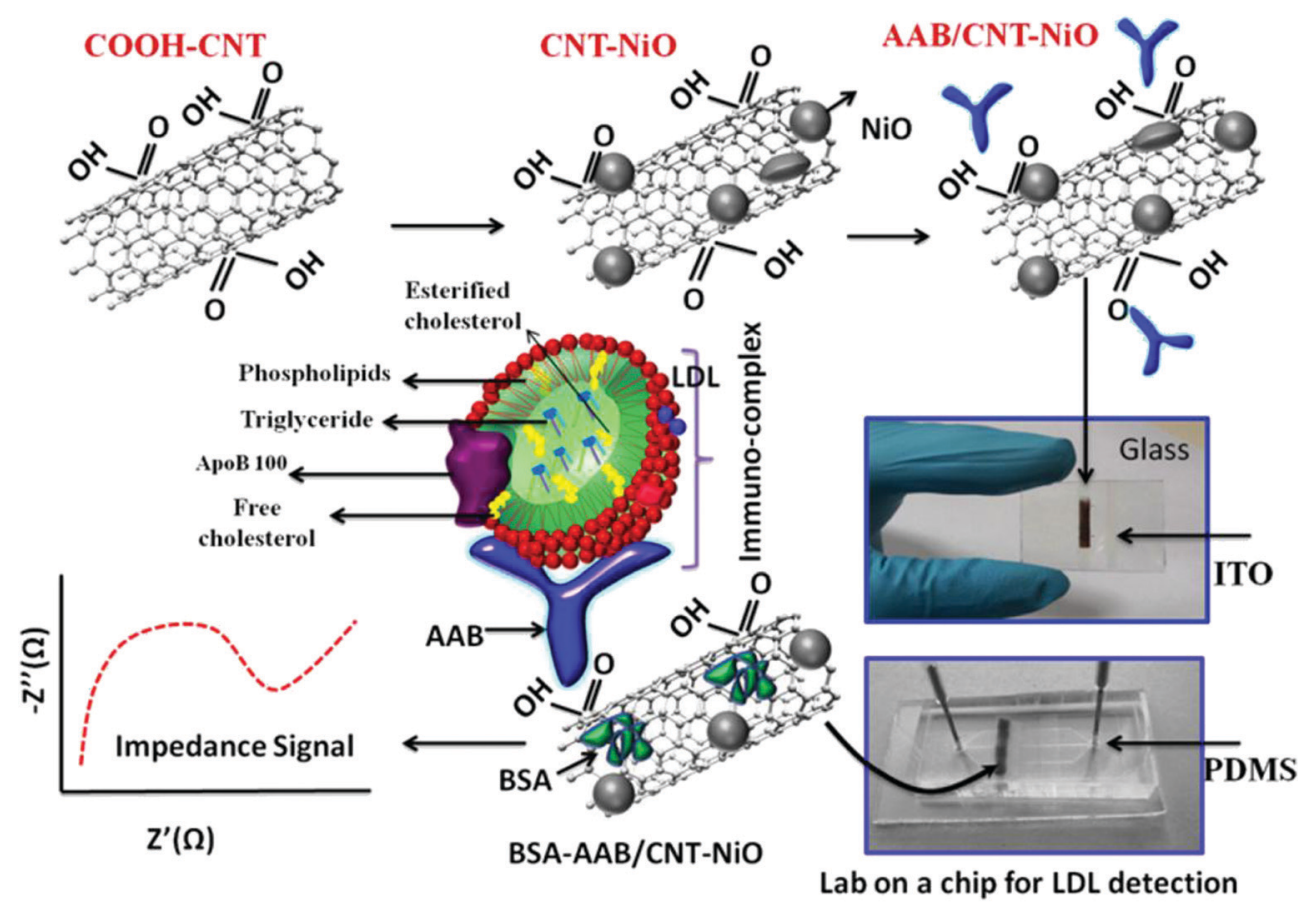

Fig. 8 Schematic representation of the lab-on-a-chip fabrication for LDL detection. Image reprinted from ref. 94 with permission. Copyright 2015 American Chemical Society.

A prominent application of AFNs is cell targeting. There are numerous aptamers currently available for various cellular targets. Targets can be cell-surface binding proteins, viruses, etc. With nanomaterials as drug carriers, they can be used together with detection instruments or devices, such as fluorescence. Nanomaterials can improve drug stability and reduce toxicity and side effects. At present, the development of a multi-functional nanoplatform has been used in biomedical research. ${ }^{104,105}$ Aptamerguided nanomedicine shows great promise without affecting the homeostasis of the physiological environment or the integrity of the nearby tissue in targeted cancer therapies. In the case of delivery, nanoparticle-based carrier systems have a unique ability to cross biological barriers. Therefore, nanoparticles can leak into the tumor through its localized blood vessels and be retained due to poor lymphatic drainage in the tumor microenvironment. Tan et al. reported a study on how the physiological environment affects targeting to investigate the loss of such targeting. Aptamerfunctionalized AuNPs were selected as models and exposed to human serum to simulate the physiological environment. ${ }^{106}$ Liposomes are considered to be one of the most successful nanoplatforms for drug delivery due to their similarities with the structure and function of cell membranes, and have been used to transfer a variety of small molecules, genes, and even nanoparticles. ${ }^{107,108}$ MSN is a kind of surface porous structure material. When MSN is used as a carrier, internally packaged drugs are released by using different pore inhibitors and respond to different stimuli, including $\mathrm{pH}$, temperature, light, enzyme and competitive binding. ${ }^{109,110}$ Aptamer-modified fluorescein molecules or other materials can be applied to imaging localization, and the aptamer can be used for therapy. Because of the advantages of non-toxicity, rapid targeting and rapid diffusion through blood circulation, this can increase the certainty of diagnosis, treatment and clinical analysis. Furthermore, AFNs have been widely used in tumor therapy and have attracted extensive attention. Different approaches, including chemotherapy, photodynamic therapy, gene therapy and immunotherapy, have been reported in terms of therapy. Shen et al. presented the aptamer-functionalized $\mathrm{Fe}_{3} \mathrm{O}_{4} @$ carbon@doxorubicin NPs (Apt- $\left.\mathrm{Fe}_{3} \mathrm{O}_{4} @ \mathrm{C} @ D O X\right)$, and its application results in cancer synergistic chemotherapy (PTT). ${ }^{111}$ It has a strong ability to transform $808 \mathrm{~nm}$ NIR light into heat, according to energy spectrum analysis of drug release, meaning that the aptamers act as markers that allow the cancer cells to target DOX to attack a chemotherapy drug. The diversity of aptamer targets and the ease of modification are thought to make this strategy attractive for cell-based delivery and therapy. Chen et al. developed a novel aptamer-targeted light-response drug delivery system, namely the non-covalent assembly of the cy5.5-as1411 aptamer on the surface of GO coated doxorubicin loaded mesoporous silica nanoparticles (MSN-Dox@GO-Apt) for light-mediated drug release and aptamertargeted cancer therapy. ${ }^{112}$ The two switches of MSN-Dox@GO-Apt are respectively controlled by the appropriate body targeting and light triggering. The Cy5.5-AS1411 ligand provides MSN-Dox@GOApt with nucleoside specific targeting and real-time indication by turning off the Cy5.5 fluorescence recovery function. In the absence of laser light, GO acts as a gatekeeper, preventing the load of GO from leaking, and controlling its release under laser light. When the GO coating falls off under laser light, the "switch" photosensitive drug delivery system is activated, inducing chemotherapy. Interestingly, with increased laser power, the synergistic effect of chemotherapy and photothermal therapy on a single 


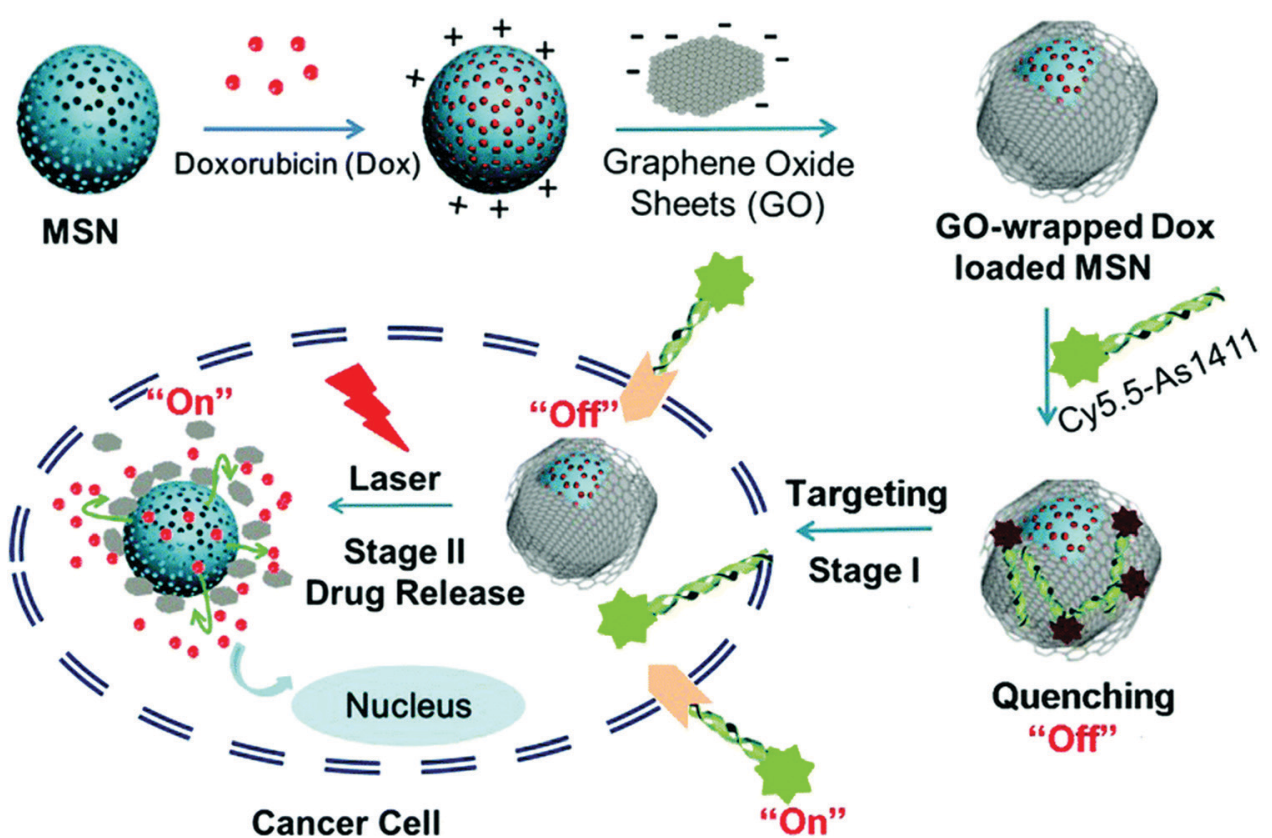

Fig. 9 Schematic illustration of GO-wrapped DOX-loaded MSN-NH 2 bound with Cy5.5-labeled AS1411 aptamer and the corresponding NIR lightcontrolled intracellular drug release. The two "off-on" switches of MSN-Dox@GO-Apt were controlled by aptamer targeting and light triggering, respectively. Image reprinted from ref. 112 with permission. Copyright 2015 Royal Society of Chemistry.

MSN-DOX@GO-Apt platform was more effective than monotherapy in killing cancer cells, providing a new approach to cancer treatment (Fig. 9).

However, despite many exciting and dramatic developments emerging at the moment, these functional nanomaterials still face many challenges in the further clinical application of cancer therapy. One of the most important issues is the potential long-term safety of nanomaterials. In particular, nonbiodegradable inorganic materials remain in the body for a long time after administration. In addition, the future of cancer treatment is likely to rely on the combination of a series of different treatments, including surgery, chemotherapy, radiation therapy, gene therapy, as well as heat and light power therapy. As a result, the development of multi-functional nanocarriers that can combine different therapeutic mechanisms to treat tumors brings great opportunities for a new generation of cancer therapies.

\subsection{Chemiluminescence}

Aptamer-functional nanomaterials with chemiluminescence properties have attracted increasing attention from researchers due to their excellent chemiluminescence properties, good stability and reproducibility. Currently, due to the incomplete recognition of the binding site and conformation change of the target ligand after binding, it is difficult to observe the marker site, and even labeling or modification reduces the binding affinity between the target and its adapter, thus reducing sensitivity and creating other problems. Facing this kind of challenge, chemiluminescence (CL) has attracted the attention of many researchers.

CL is an effective and powerful analytical technique with extremely high sensitivity and other advantages, such as simple instrumentation. CL does not need an external light source to generate a signal. In fluorescent assays, aptamers can serve as recognition moieties and control the distance between quenchers. In recent years, a few aptamer-based sensing platforms have been proposed to use in CL detection. For example, Li et al. reported a label-free, aptamer-based chemiluminescent biosensor, which relies upon the catalytic activity of unmodified AuNPs on the luminol- $\mathrm{H}_{2} \mathrm{O}_{2}$ CL reaction, and the interaction of unmodified AuNPs with the aptamer. ${ }^{113}$ Wang et al. developed a highly specific and sensitive CL aptamer sensor for sulfamethazine (SMZ) by taking advantage of the supernormal selectivity of a new selected aptamer. ${ }^{114}$ Finally, the aptamer sensor was applied to milk samples, and the accuracy was better. In addition, AS1411 is a versatile bioassay aptamer, which has been applied to the chemiluminescence detection of nucleosides on the surface of HeLa cells. In view of this condition, our group previously carried out a study and proposed a new method to prepare AS1411 G-quadruplex and folic acid-double-targeted fluorescent gold nanocomposite (AF-D-AuNP) with DOX. ${ }^{104}$ In this study, AF-DAuNP cover support AS1411 was successfully developed and shows significant potential in the early detection of cancer due to its high light bleaching. Sancenón and co-workers developed a novel sensing nanometer probe for the selective sensitive detection of As(III) based on the combination of aptamer and MSN. ${ }^{115}$ The sensing mechanism depends on the specific interaction between the capped-shaped aptamer (ARS-3) and As(III). The efficiency of the sensor has been verified under environmental conditions, showing great potential in As(III) monitoring. The prepared nanoprobe showed a remarkable limit of detection of 0.9 PPB. The authors believe that the method is simple and easy to use and has potential applications (Fig. 10). 


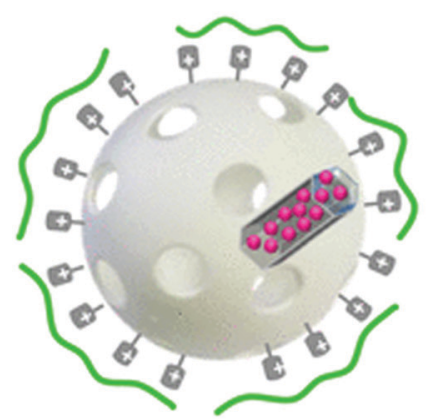

S1-Ars-3
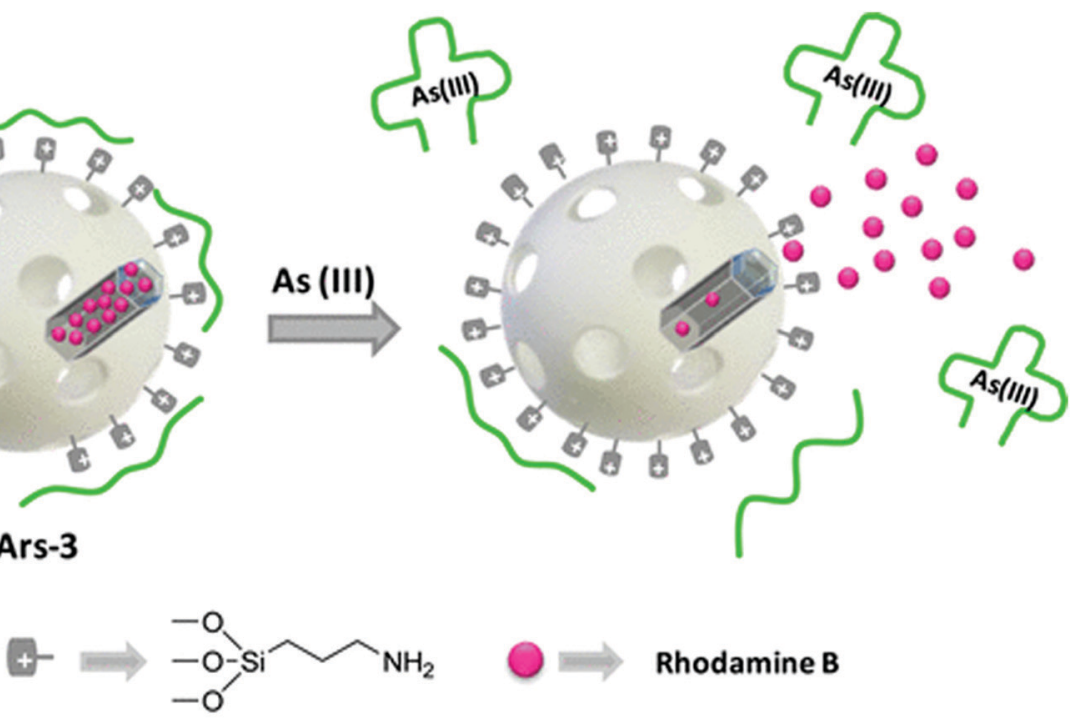

\section{Ars-3: 5'- GGT AAT ACG ACT CAC TAT AGG GAG ATA CCA GCT \\ TAT TCA ATT TTA CAG AAC AAC CAA CGT CGC TCC GGG TAC TTC TTC ATC GAG ATA GTA AGT GCA ATCT - 3 '}

Fig. 10 Representation of the gated material S1-Ars-3 functionalized with aminopropyl moieties and capped with Ars-3 aptamer. Delivery of the entrapped dye (rhodamine B) is specifically accomplished in the presence of As(III). Image reprinted from ref. 114 with permission. Copyright 2014 American Chemical Society.

\section{Conclusions and perspective}

An aptamer is an oligonucleotide sequence that can be specifically bound to a target object, easily synthesized in vitro, and modified by various simple chemicals. Because the aptamers are non-immunogenic and non-toxic, and the types of target aptamers are not restricted, they can be used in the diagnosis and treatment of diseases in the field of biological applications. Based on all these advantages, AFNs have become increasingly important molecular tools for diagnosis and treatment. In this review, we discuss the development of AFNs in catalytic, biosensing, imaging, targeted drug delivery and cancer cell treatment. Despite the many exciting and compelling developments that are emerging at the moment, several challenges and important issues still need to be addressed further: (1) to develop further synthetic strategies for the preparation of AFNs. Since the immobilization of the aptamer may interfere with its combined properties, the aptamer density, aptamer orientation, surface charge and steric hindrance should be considered. (2) To improve efficiency via optimizing the AFN. Its main purpose is to solve the problem of optimal binding performance after the aptamer is fixed on the carrier. (3) To explore novel physical-chemical properties of AFN structures. Further consider combining materials of different dimensions (one-, two-, three-) to construct unique properties. (4) To promote the development of practical applications of AFNs; for example, to solve the problem of toxicity of AFNs. So far, various efficient and practical AFN engineering technologies have been developed. These nanoscale devices have proven to be ideal tools for various biological applications, with good biocompatibility, high endocytosis efficiency and high affinity for target analytes. In the future development of nanomaterials, increasing attention will be paid to the preparation of new nanomaterials and the improvement of aptamer properties.

\section{Conflicts of interest}

There are no conflicts to declare.

\section{Acknowledgements}

This work was supported by the National Natural Science Foundation of China (Grant No. 21864020, 21925405), the Natural Science Foundation of Inner Mongolia (Grant No. 2018MS02012 and 2019MS02014), Collaborative Innovation Center for Water Environmental Security of Inner Mongolia Autonomous Region, China (Grant no. XTCX003), "Young Science and Technology Talents Program" (Leading Person) in Inner Mongolia Autonomous Region Colleges and Universities (Grant no. NJYT-19-A04).

\section{References}

1 C. M. Niemeyer, Nanoparticles, proteins, and nucleic acids: biotechnology meets materials science, Angew. Chem., Int. Ed., 2001, 40, 4128-4158.

2 G. M. Clore and A. M. Gronenborn, Determining the structures of large proteins and protein complexes by NMR, Trends Biotechnol., 1998, 16, 22-34. 
3 H. Shirai, A. Kidera and H. Nakamura, H3-rules: identification of CDR-H3 structures in antibodies, FEBS Lett., 1999, 455, 188-197.

$4 \mathrm{~N}$. Yathindra and M. Sundaralingam, Analysis of the possible helical structures of nucleic acids and polynucleotides. Application of (nh) plots, Nucleic Acids Res., 1976, 3, 729-748.

5 Y. Sone, R. Okuda, N. Wada, E. Kishida and A. Misaki, Structures and antitumor activities of the polysaccharides isolated from fruiting body and the growing culture of mycelium of Ganoderma lucidum, Agric. Biol. Chem., 1985, 49, 2641-2653.

6 A. D. Ellington and J. W. Szostak, In vitro selection of RNA molecules that bind specific ligands, Nature, 1990, 346, 818-822.

7 C. Tuerk and L. Gold, Systematic evolution of ligands by exponential enrichment: RNA ligands to bacteriophage T4 DNA polymerase, Science, 1990, 249, 505-510.

8 C. H. Alyssa and H. Jonathan, High-order structures from nucleic acids for biomedical applications, Mater. Chem. Front, 2020, DOI: 10.1039/C9QM00638A.

9 O. N. Oliveira Jr, R. M. Iost, J. R. Siqueira Jr, F. N. Crespilho and L. Caseli, Nanomaterials for diagnosis: challenges and applications in smart devices based on molecular recognition, ACS Appl. Mater. Interfaces, 2014, 6, 14745-14766.

10 M. ChingáCheung, H. ManáLeung, T. C. KongáLau, K. W. ChiuáLai and P. KwanáLo, A highly versatile platform based on geometrically well-defined 3D DNA nanostructures for selective recognition and positioning of multiplex targets, Nanoscale, 2016, 8, 18291-18295.

11 K. H. Bae, H. J. Chung and T. G. Park, Nanomaterials for cancer therapy and imaging, Mol. Cells, 2011, 31, 295-302.

12 S. Zaib and J. Iqbal, Nanotechnology: Applications, techniques, approaches, \& the advancement in toxicology and environmental impact of engineered nanomaterials, Importance \& Applications of Nanotechnology, 2019.

13 N. L. Rosi and C. A. Mirkin, Nanostructures in biodiagnostics, Chem. Rev., 2005, 105, 1547-1562.

14 Z. Liu, J. T. Robinson, X. Sun and H. Dai, PEGylated nanographene oxide for delivery of water-insoluble cancer drugs, J. Am. Chem. Soc., 2008, 130, 10876-10877.

15 C. Cheng, S. Li, A. Thomas, N. A. Kotov and R. Haag, Functional graphene nanomaterials based architectures: biointeractions, fabrications, and emerging biological applications, Chem. Rev., 2017, 117, 1826-1914.

16 L. Ye, K. T. Yong, L. W. Liu, I. Roy, R. Hu, J. Zhu, H. X. Cai, W. C. Law, J. W. Liu, K. Wang, J. Liu, Y. Q. Liu, Y. Z. Hu, X. H. Zhang, M. T. Swihart and P. N. Prasad, A pilot study in nonhuman primates shows no adverse response to intravenous injection of quantum dots, Nat. Nanotechnol., 2012, 7, 453-458.

17 L. Yan, F. Zhao, J. Wang, Y. Zu, Z. Gu and Y. Zhao, A Safeby-Design Strategy towards Safer Nanomaterials in Nanomedicines, Adv. Mater., 2019, 1805391.

18 S. Chakraborty, Z. Y. Dlie, B. Mukherjee, S. E. Besra, S. Sengupta, R. Sen and A. Mukherjee, A Comparative
Investigation of the Ability of Various AptamerFunctionalized Drug Nanocarriers to Induce Selective Apoptosis in Neoplastic Hepatocytes: In Vitro and In Vivo Outcome, AAPS PharmSciTech, 2020, 21, 89.

19 S. Chakraborty, Z. Dile, S. Chakraborty, S. Roy, B. Mukherjee, S. E. Besra and R. Sen, Aptamer-functionalized drug-nanocarrier improves hepatocellular carcinoma towards normal by targeting neoplastic hepatocytes, Mol. Ther.-Nucleic Acids, 2020, 20, 34-49.

20 H. Wang, R. Yang, L. Yang and W. Tan, Nucleic acid conjugated nanomaterials for enhanced molecular recognition, ACS Nano, 2009, 3, 2451-2460.

21 Y. Liu, X. Ji and Z. He, Organic-inorganic nanoflowers: from design strategy to biomedical applications, Nanoscale, 2019, 11, 17179-17194.

22 L. Li, H. Xing, J. Zhang and Y. Lu, Functional DNA Molecules Enable Selective and Stimuli-Responsive Nanoparticles for Biomedical Applications, Acc. Chem. Res., 2019, 52, 2415-2426.

23 J. J. Cha, H. Lee, M. Kim, J. Kang, H. Song, M. G. Kim and J. H. Lee, Immobilized DNA aptamers used as potent attractors for vascular endothelial cell: in vitro study of female rat, Biosci. Rep., 2020, 40, BSR20182444.

24 H. Elmizadeh, F. Faridbod, M. Soleimani, M. R. Ganjali and G. R. Bardajee, Fluorescent apta-nanobiosensors for fast and sensitive detection of digoxin in biological fluids using rGQDs: Comparison of two approaches for immobilization of aptamer, Sens. Actuators, B, 2020, 302, 127133.

25 A. Bolotsky, D. Butler, C. Dong, K. Gerace, N. R. Glavin, C. Muratore and A. Ebrahimi, Two-Dimensional Materials in Biosensing and Healthcare: From In Vitro Diagnostics to Optogenetics and Beyond, ACS Nano, 2019, 13, 9781-9810.

26 A. Biswas, A. Shukla and P. Maiti, Biomaterials for Interfacing Cell Imaging and Drug Delivery: An Overview, Langmuir, 2019, 35, 12285-12305.

27 R. Conte, A. Valentino, F. Di Cristo, G. Peluso, P. Cerruti, A. Di Salle and A. Calarco, Cationic Polymer NanoparticlesMediated Delivery of miR-124 Impairs Tumorigenicity of Prostate Cancer Cells, Int. J. Mol. Sci., 2020, 21, 869.

28 S. Pengnam, S. Plainwong, P. Patrojanasophon, T. Rojanarata, T. Ngawhirunpat, W. Radchatawedchakoon and P. Opanasopit, Effect of hydrophobic tails of plier-like cationic lipids on nucleic acid delivery and intracellular trafficking, Int. J. Pharm., 2020, 573, 118798.

29 M. V. Yigit, D. Mazumdar, H. K. Kim, J. H. Lee, B. Odintsov and Y. Lu, Smart "turn-on" magnetic resonance contrast agents based on aptamer-functionalized superparamagnetic iron oxide nanoparticles, ChemBioChem, 2007, 8, 1675-1678.

30 X. Zhao, R. Tapec-Dytioco, K. Wang and W. Tan, Collection of trace amounts of DNA/mRNA molecules using genomagnetic nanocapturers, Anal. Chem., 2003, 75, 3476-3483.

31 O. Veiseh, J. W. Gunn and M. Zhang, Design and fabrication of magnetic nanoparticles for targeted drug delivery and imaging, Adv. Drug Delivery Rev., 2010, 62, 284-304.

32 X. Huang, I. H. El-Sayed, W. Qian and M. A. El-Sayed, Cancer cells assemble and align gold nanorods conjugated 
to antibodies to produce highly enhanced, sharp, and polarized surface Raman spectra: a potential cancer diagnostic marker, Nano Lett., 2007, 7, 1591-1597.

33 G. Liu, X. Mao, J. A. Phillips, H. Xu, W. Tan and L. Zeng, Aptamer-nanoparticle strip biosensor for sensitive detection of cancer cells, Anal. Chem., 2009, 81, 10013-10018.

34 S. H. Cheng, C. H. Lee, C. S. Yang, F. G. Tseng, C. Y. Mou and L. W. Lo, Mesoporous silica nanoparticles functionalized with an oxygen-sensing probe for cell photodynamic therapy: potential cancer theranostics, J. Mater. Chem., 2009, 19, 1252-1257.

35 L. R. Hilliard, X. Zhao and W. Tan, Immobilization of oligonucleotides onto silica nanoparticles for DNA hybridization studies, Anal. Chim. Acta, 2002, 470, 51-56.

36 X. He, B. Wei and Y. Mi, Aptamer based reversible DNA induced hydrogel system for molecular recognition and separation, Chem. Commun., 2010, 46, 6308-6310.

37 Z. Zhu, C. Wu, H. Liu, Y. Zou, X. Zhang, H. Kang and W. Tan, An aptamer cross-linked hydrogel as a colorimetric platform for visual detection, Angew. Chem., Int. Ed., 2010, 49, 1052-1056.

38 H. M. So, D. W. Park, H. Chang and J. O. Lee, Carbon nanotube biosensors with aptamers as molecular recognition elements, Carbon Nanotubes, Humana Press, 2010, pp. 239-249.

39 R. Yang, J. Jin, Y. Chen, N. Shao, H. Kang, Z. Xiao and W. Tan, Carbon nanotube-quenched fluorescent oligonucleotides: probes that fluoresce upon hybridization, J. Am. Chem. Soc., 2008, 130, 8351-8358.

40 M. J. O'connell, S. M. Bachilo, C. B. Huffman, V. C. Moore, M. S. Strano, E. H. Haroz and J. Ma, Band gap fluorescence from individual single-walled carbon nanotubes, Science, 2002, 297, 593-596.

41 N. Yu, X. Zhang, Y. Gao, H. You, J. Zhang and P. Miao, Highly Sensitive Endotoxin Assay Combining Peptide/ Graphene Oxide and DNA-Modified Gold Nanoparticles, ACS Omega, 2019, 4, 14312-14316.

42 S. Yousefi and M. Saraji, Optical aptasensor based on silver nanoparticles for the colorimetric detection of adenosine, Spectrochim. Acta, Part A, 2019, 213, 1-5.

43 C. C. Chang, C. P. Chen, T. H. Wu, C. H. Yang, C. W. Lin and C. Y. Chen, Gold Nanoparticle-Based Colorimetric Strategies for Chemical and Biological Sensing Applications, Nanomaterials, 2019, 9, 861.

44 N. Zhou, F. Su, Z. Li, X. Yan, C. Zhang, B. Hu and Z. Zhang, Gold nanoparticles conjugated to bimetallic manganese (II) and iron(II) Prussian Blue analogues for aptamer-based impedimetric determination of the human epidermal growth factor receptor-2 and living MCF-7 cells, Microchim. Acta, 2019, 186, 75.

45 H. Xu, X. Mao, Q. Zeng, S. Wang, A. N. Kawde and G. Liu, Aptamer-functionalized gold nanoparticles as probes in a dry-reagent strip biosensor for protein analysis, Anal. Chem., 2008, 81, 669-675.

46 L. Zhu, S. Li, X. Shao, Y. Feng, P. Xie, Y. Luo and W. Xu, Colorimetric detection and typing of E. coli lipopolysaccharides based on a dual aptamer-functionalized gold nanoparticle probe, Microchim. Acta, 2019, 186, 111.

47 Y. Ou, X. Jin, J. Liu, Y. Tian and N. Zhou, Visual detection of kanamycin with DNA-functionalized gold nanoparticles probe in aptamer-based strip biosensor, Anal. Biochem., 2019, 587, 113432.

48 V. Pavlov, Y. Xiao, B. Shlyahovsky and I. Willner, Aptamerfunctionalized $\mathrm{Au}$ nanoparticles for the amplified optical detection of thrombin, J. Am. Chem. Soc., 2004, 126, 11768-11769.

49 Y. Gan, T. Liang, Q. Hu, L. Zhong, X. Wang, H. Wan and P. Wang, In-situ detection of cadmium with aptamer functionalized gold nanoparticles based on smartphonebased colorimetric system, Talanta, 2020, 208, 120231.

50 X. He, Y. Zhao, D. He, K. Wang, F. Xu and J. Tang, ATPresponsive controlled release system using aptamerfunctionalized mesoporous silica nanoparticles, Langmuir, 2012, 28, 12909-12915.

51 R. Sakhtianchi, B. Darvishi, Z. Mirzaie, F. Dorkoosh, S. Shanehsazzadeh and R. Dinarvand, Pegylated magnetic mesoporous silica nanoparticles decorated with AS1411 Aptamer as a targeting delivery system for cytotoxic agents, Pharm. Dev. Technol., 2019, 1-36.

52 S. E. Harton, S. K. Kumar, H. Yang, T. Koga, K. Hicks, H. Lee and D. W. Gidley, Immobilized polymer layers on spherical nanoparticles, Macromolecules, 2010, 43, 3415-3421.

53 P. A. Fiorito, V. R. Gonçales, E. A. Ponzio and S. I. C. de Torresi, Synthesis, characterization and immobilization of Prussian blue nanoparticles. A potential tool for biosensing devices, Chem. Commun., 2005, 366-368.

$54 \mathrm{~J}$. Liu and Y. Lu, Adenosine-dependent assembly of aptazymefunctionalized gold nanoparticles and its application as a colorimetric biosensor, Anal. Chem., 2004, 76, 1627-1632.

55 M. Xiao, W. Lai, T. Man, B. Chang, L. Li, A. R. Chandrasekaran and H. Pei, Rationally Engineered Nucleic Acid Architectures for Biosensing Applications, Chem. Rev., 2019, 119, 11631-11717.

56 V. Pavlov, Y. Xiao, B. Shlyahovsky and I. Willner, Aptamerfunctionalized $\mathrm{Au}$ nanoparticles for the amplified optical detection of thrombin, J. Am. Chem. Soc., 2004, 126, 11768-11769.

57 F. Li, J. Li, C. Wang, J. Zhang, X. F. Li and X. C. Le, Competitive protection of aptamer-functionalized gold nanoparticles by controlling the DNA assembly, Anal. Chem., 2011, 83, 6464-6467.

58 M. I. Shukoor, M. O. Altman, D. Han, A. T. Bayrac, I. Ocsoy, Z. Zhu and W. Tan, Aptamer-nanoparticle assembly for logic-based detection, ACS Appl. Mater. Interfaces, 2012, 4, 3007-3011.

59 L. Zhao, D. Kong, Z. Wu, G. Liu, Y. Gao, X. Yan and G. Lu, Interface interaction of $\mathrm{MoS}_{2}$ nanosheets with DNA based aptameric biosensor for carbohydrate antigen 15-3 detection., Microchem. J., 2020, 104675.

60 R. Orbach, W. Guo, F. Wang, O. Lioubashevski and I. Willner, Self-assembly of luminescent $\mathrm{Ag}$ nanocluster-functionalized nanowires, Langmuir, 2013, 29, 13066-13071. 
61 X. Ma, J. Huh, W. Park, L. P. Lee, Y. J. Kwon and S. J. Sim, Gold nanocrystals with DNA-directed morphologies, Nat. Commun., 2016, 7, 12873.

62 T. Qing, B. Feng, P. Zhang, K. Zhang, X. He and K. Wang, Beyond Native Deoxyribonucleic Acid, Templating Fluorescent Nanomaterials for Bioanalytical Applications: A Review, Anal. Chim. Acta, 2020, 1105, 11-27.

63 K. Y. Goud, K. K. Reddy, M. Satyanarayana, S. Kummari and K. V. Gobi, A review on recent developments in optical and electrochemical aptamer-based assays for mycotoxins using advanced nanomaterials, Microchim. Acta, 2020, 187, 29.

64 Q. L. Wang, W. X. Huang, P. J. Zhang, L. Chen, C. K. Lio, H. Zhou and P. Luo, Colorimetric determination of the early biomarker hypoxia-inducible factor-1 alpha (HIF-1 $\alpha$ ) in circulating exosomes by using a gold seed-coated with aptamer-functionalized $\mathrm{Au} @ \mathrm{Au}$ core-shell peroxidase mimic, Microchim. Acta, 2020, 187, 61.

65 M. Madsen and K. V. Gothelf, Chemistries for DNA Nanotechnology, Chem. Rev., 2019, 119, 6384-6458.

66 A. B. Gupta, S. Mukherjee, C. Q. Pan, A. Velazquez-Campoy, J. Sivaraman and B. C. Low, Spatial arrangement of LD motif-interacting residues on focal adhesion targeting domain of Focal Adhesion Kinase determine domainmotif interaction affinity and specificity, Biochim. Biophys. Acta, Gen. Subj., 1864, 2020, 129450.

67 T. Li, L. Zhang, J. Ai, S. Dong and E. Wang, Ion-tuned DNA/ $\mathrm{Ag}$ fluorescent nanoclusters as versatile logic device, ACS Nano, 2011, 5, 6334-6338.

68 H. Huang, S. Shi, X. Gao, R. Gao, Y. Zhu, X. Wu and T. Yao, A universal label-free fluorescent aptasensor based on $\mathrm{Ru}$ complex and quantum dots for adenosine, dopamine and $17 \beta$-estradiol detection, Biosens. Bioelectron., 2016, 79, 198-204.

69 M. Yaseen, F. Pan, X. Zhao and J. R. Lu, Surface modification to improve biocompatibility, Comprehensive Biotechnology, Elsevier BV, 2019, pp. 471-487.

$70 \mathrm{~T}$. Chandy, Biocompatibility of materials and its relevance to drug delivery and tissue engineering, Biointegration of Medical Implant Materials, Woodhead Publishing, 2020.

71 A. Gothwal, S. Malik, U. Gupta and N. K. Jain, Toxicity and biocompatibility aspects of dendrimers, Pharmaceutical Applications of Dendrimers, Elsevier, 2020.

72 M. Centola, J. Valero and M. Famulok, Allosteric control of oxidative catalysis by a DNA rotaxane nanostructure, J. Am. Chem. Soc., 2017, 139, 16044-16047.

73 F. Li, Q. Gao, M. Yang and W. Guo, Regulation of Catalytic DNA Activities with Thermosensitive Gold Nanoparticle Surfaces, Langmuir, 2018, 34, 14932-14939.

74 F. Li, C. Wang and W. Guo, Multifunctional Poly-NIsopropylacrylamide/DNAzyme Microgels as Highly Efficient and Recyclable Catalysts for Biosensing, Adv. Funct. Mater., 2018, 28, 1705876.

75 C. W. Wu, S. G. Harroun, C. W. Lien, H. T. Chang, B. Unnikrishnan, I. P. J. Lai and C. C. Huang, Selftemplated formation of aptamer-functionalized copper oxide nanorods with intrinsic peroxidase catalytic activity for protein and tumor cell detection, Sens. Actuators, B, 2016, 227, 100-107.

76 J. Ai, W. Guo, B. Li, T. Li, D. Li and E. Wang, DNA G-quadruplex-templated formation of the fluorescent silver nanocluster and its application to bioimaging, Talanta, 2012, 88, 450-455.

77 J. Ai, Y. Xu, D. Li, Z. Liu and E. Wang, Folic acid as delivery vehicles: targeting folate conjugated fluorescent nanoparticles to tumors imaging, Talanta, 2012, 101, 32-37.

78 J. Ai, J. Li, L. Ga, G. Yun, L. Xu and E. Wang, Multifunctional near-infrared fluorescent nanoclusters for simultaneous targeted cancer imaging and photodynamic therapy, Sens. Actuators, B, 2016, 222, 918-922.

79 P. Peng, Y. Du, J. Zheng, H. Wang and T. Li, Reconfigurable Bioinspired Framework Nucleic Acid Nanoplatform Dynamically Manipulated in Living Cells for Subcellular Imaging, Angew. Chem., Int. Ed., 2019, 58, 1648-1653.

80 Y. Du, P. Peng and T. Li, DNA Logic Operations in Living Cells Utilizing Lysosome-Recognizing Framework Nucleic Acid Nanodevices for Subcellular Imaging, ACS Nano, 2019, 13, 5778-5784.

81 J. Li, X. Zhong, F. Cheng, J. R. Zhang, L. P. Jiang and J. J. Zhu, One-pot synthesis of aptamer-functionalized silver nanoclusters for cell-type-specific imaging, Anal. Chem., 2012, 84, 4140-4146.

82 Y. Zhang, M. Leonard, Y. Shu, Y. Yang, D. Shu, P. Guo and $\mathrm{X}$. Zhang, Overcoming tamoxifen resistance of human breast cancer by targeted gene silencing using multifunctional pRNA nanoparticles, ACS Nano, 2016, 11, 335-346.

83 H. Xu, X. Mao, Q. Zeng, S. Wang, A. N. Kawde and G. Liu, Aptamer-functionalized gold nanoparticles as probes in a dry-reagent strip biosensor for protein analysis, Anal. Chem., 2009, 81, 669-675.

84 Y. Gan, T. Liang, Q. Hu, L. Zhong, X. Wang, H. Wan and P. Wang, In-situ detection of cadmium with aptamer functionalized gold nanoparticles based on smartphonebased colorimetric system, Talanta, 2020, 208, 120231.

85 M. Chen, M. Hassan, H. Li and Q. Chen, Fluorometric determination of lead(II) by using aptamer-functionalized upconversion nanoparticles and magnetite-modified gold nanoparticles, Microchim. Acta, 2020, 187, 1-9.

86 T. C. Chiu and C. C. Huang, Aptamer-functionalized nanobiosensors, Sensors, 2009, 9, 10356-10388.

87 J. Wang, S. Mao, H. F. Li and J. M. Lin, Multi-DNAzymesfunctionalized gold nanoparticles for ultrasensitive chemiluminescence detection of thrombin on microchip, Anal. Chim. Acta, 2018, 1027, 76-82.

88 W. H. Wu, M. Li, Y. Wang, H. X. Ouyang, L. Wang, C. X. Li and J. X. Lu, Aptasensors for rapid detection of Escherichia coli O157: H7 and Salmonella typhimurium, Nanoscale Res. Lett., 2012, 7, 658.

89 E. Bagheri, K. Abnous, M. Alibolandi, M. Ramezani and S. M. Taghdisi, Triple-helix molecular switch-based aptasensors and DNA sensors, Biosens. Bioelectron., 2018, 111, 1-9. 
90 M. Liu, A. Khan, Z. Wang, Y. Liu, G. Yang, Y. Deng and N. He, Aptasensors for pesticide detection, Biosens. Bioelectron., 2019, 130, 174-184.

91 R. Bala, S. Dhingra, M. Kumar, K. Bansal, S. Mittal, R. K. Sharma and N. Wangoo, Detection of organophosphorus pesticide-malathion in environmental samples using peptide and aptamer based nanoprobes, Chem. Eng. J., 2017, 311, 111-116.

92 N. Wongkaew, M. Simsek, C. Griesche and A. J. Baeumner, Functional nanomaterials and nanostructures enhancing electrochemical biosensors and lab-on-a-chip performances: Recent progress, applications, and future perspective, Chem. Rev., 2018, 119, 120-194.

93 E. Primiceri, M. S. Chiriacò, F. de Feo, E. Santovito, V. Fusco and G. Maruccio, A multipurpose biochip for food pathogen detection, Anal. Methods, 2016, 8, 3055-3060.

94 Md. Azahar Ali, P. R. Solanki, S. Srivastava, S. Singh, V. V. Agrawal, R. John and B. D. Malhotra, Protein Functionalized Carbon Nanotubes-based Smart Lab-on-a-Chip, ACS Appl. Mater. Interfaces, 2015, 7(10), 5837-5846.

95 M. Healy Judith, D. Lewis Scott, K. Markus, M. Boomer Ryan, M. Thompson Kristin, W. Charles and G. McCauley Thomas, Pharmacokinetics and biodistribution of novel aptamer compositions, Pharm. Res., 2004, 21, 2234-2246.

96 A. K. Croker and A. L. Allan, Cancer stem cells: implications for the progression and treatment of metastatic disease, J. Cell. Mol. Med., 2008, 12, 374-390.

97 P. R. Bouchard, R. M. Hutabarat and K. M. Thompson, Discovery and development of therapeutic aptamers, Annu. Rev. Pharmacol. Toxicol., 2010, 50, 237-257.

98 A. D. Keefe and S. T. Cload, SELEX with modified nucleotides, Curr. Opin. Chem. Biol., 2008, 12, 448-456.

99 C. Wilson, Aptamer opportunities and challenges, Antisense Drug Technology, 2007, pp. 791-818.

100 P. R. Bouchard, R. M. Hutabarat and K. M. Thompson, Discovery and development of therapeutic aptamers, Annu. Rev. Pharmacol. Toxicol., 2010, 50, 237-257.

101 M. McKeague and M. C. DeRosa, Challenges and opportunities for small molecule aptamer development, J. Nucleic Acids, 2012, 2012, 748913.

102 J. Zhou and J. Rossi, Aptamers as targeted therapeutics: current potential and challenges, Nat. Rev. Drug Discovery, 2017, 16(3), 181.

103 H. Kaur, J. G. Bruno, A. Kumar and T. K. Sharma, Aptamers in the therapeutics and diagnostics pipelines, Theranostics, 2018, 8, 4016.
104 J. Ai, L. Ga and Y. Wang, A dual-targeting AS1411-folic acid fluorescent nanocomposite for cancer cell and drug delivery, Anal. Methods, 2018, 10, 1949-1951.

105 J. Ai, J. Li, L. Ga, G. Yun, L. Xu and E. Wang, G-quadruplex/ protoporphyrin IX-functionalized silver nanoconjugates for targeted cancer cell photodynamic therapy, RSC Adv., 2016, 6, 96942-96945.

106 D. Ding, Y. Zhang, E. A. Sykes, L. Chen, Z. Chen and W. Tan, The influence of physiological environment on the targeting effect of aptamer-guided gold nanoparticles, Nano Res., 2019, 12, 129-135.

107 S. Tran, P. J. DeGiovanni, B. Piel and P. Rai, Cancer nanomedicine: a review of recent success in drug delivery, Clin. Transl. Sci., 2017, 6, 44.

108 H. Ragelle, F. Danhier, V. Préat, R. Langer and D. G. Anderson, Nanoparticle-based drug delivery systems: a commercial and regulatory outlook as the field matures, Expert Opin. Drug Delivery, 2017, 14, 851-864.

109 C. Reinemann and B. Strehlitz, Aptamer-modified nanoparticles and their use in cancer diagnostics and treatment, Swiss Med. Wkly., 2014, 144, 13908.

110 S. A. Moosavian, K. Abnous, A. Badiee and M. R. Jaafari, Improvement in the drug delivery and anti-tumor efficacy of PEGylated liposomal doxorubicin by targeting RNA aptamers in mice bearing breast tumor model, Colloids Surf., B, 2016, 139, 228-236.

111 C. Zhao, X. Song, W. Jin, F. Wu, Q. Zhang, M. Zhang and $\mathrm{J}$. Shen, Image-guided cancer therapy using aptamer-functionalized cross-linked magnetic-responsive $\mathrm{Fe}_{3} \mathrm{O}_{4}$ @ carbon nanoparticles, Anal. Chim. Acta, 2019, 1056, 108-116.

112 Y. Tang, H. Hu, M. G. Zhang, J. Song, L. Nie, S. Wang and $\mathrm{X}$. Chen, An aptamer-targeting photoresponsive drug delivery system using "off-on" graphene oxide wrapped mesoporous silica nanoparticles, Nanoscale, 2015, 7, 6304-6310.

113 Y. Qi and B. Li, A Sensitive, Label-Free, Aptamer-Based Biosensor Using a Gold Nanoparticle-Initiated Chemiluminescence System, Chem. - Eur. J., 2011, 17, 1642-1648.

114 L. Yang, H. Ni, C. Li, X. Zhang, K. Wen, Y. Ke and Z. Wang, Development of a highly specific chemiluminescence aptasensor for sulfamethazine detection in milk based on in vitro selected aptamers, Sens. Actuators, B, 2019, 281, 801-811.

115 M. Oroval, C. Coll, A. Bernardos, M. D. Marcos, R. MartínezMáñez, D. G. Shchukin and F. Sancenón, Selective fluorogenic sensing of As(III) using aptamer-capped nanomaterials, ACS Appl. Mater. Interfaces, 2017, 9, 11332-11336. 